\title{
Impact of phase saturation on wormhole formation in rock matrix acidizing
}

DOI:

10.1016/j.ces.2017.10.046

\section{Document Version}

Accepted author manuscript

Link to publication record in Manchester Research Explorer

\section{Citation for published version (APA):}

Babaei, M., \& Sedighi, M. (2018). Impact of phase saturation on wormhole formation in rock matrix acidizing. Chemical Engineering Science, 177, 39-52. https://doi.org/10.1016/j.ces.2017.10.046

\section{Published in:}

Chemical Engineering Science

\section{Citing this paper}

Please note that where the full-text provided on Manchester Research Explorer is the Author Accepted Manuscript or Proof version this may differ from the final Published version. If citing, it is advised that you check and use the publisher's definitive version.

\section{General rights}

Copyright and moral rights for the publications made accessible in the Research Explorer are retained by the authors and/or other copyright owners and it is a condition of accessing publications that users recognise and abide by the legal requirements associated with these rights.

\section{Takedown policy}

If you believe that this document breaches copyright please refer to the University of Manchester's Takedown Procedures [http://man.ac.uk/04Y6Bo] or contact uml.scholarlycommunications@manchester.ac.uk providing relevant details, so we can investigate your claim.

\section{OPEN ACCESS}




\section{Accepted Manuscript}

Impact of phase saturation on wormhole formation in rock matrix acidizing

Masoud Babaei, Majid Sedighi

PII:

S0009-2509(17)30662-0

DOI: https://doi.org/10.1016/j.ces.2017.10.046

Reference: CES 13878

To appear in:

Chemical Engineering Science

Received Date:

12 July 2017

Revised Date:

12 October 2017

Accepted Date:

29 October 2017

Please cite this article as: M. Babaei, M. Sedighi, Impact of phase saturation on wormhole formation in rock matrix acidizing, Chemical Engineering Science (2017), doi: https://doi.org/10.1016/j.ces.2017.10.046

This is a PDF file of an unedited manuscript that has been accepted for publication. As a service to our customers we are providing this early version of the manuscript. The manuscript will undergo copyediting, typesetting, and review of the resulting proof before it is published in its final form. Please note that during the production process errors may be discovered which could affect the content, and all legal disclaimers that apply to the journal pertain. 


\title{
Impact of phase saturation on wormhole formation in rock matrix acidizing
}

\author{
Masoud Babaei ${ }^{11}$ and Majid Sedighi ${ }^{2}$ \\ ${ }^{1}$ School of Chemical Engineering and Analytical Science, The University of Manchester, M13 9PL, Manchester, \\ UK \\ ${ }^{2}$ School of Mechanical, Aerospace and Civil Engineering, The University of Manchester, M13 9PL, Manchester, \\ UK
}

\begin{abstract}
Studies of the rock matrix acidizing for enhanced recovery of oil or gas have entirely focused on the fully water saturated conditions. In fact, matrix acidizing can be conducted in low-water-cut oilproduction wells without pre-flushing of water or in high water production by injecting gas or oil ahead of the acid injection. These conditions yield a multiphase system, where the dynamics of acid transport and reactions can be altered by the presence of an immiscible phase. Against this backdrop, we present an investigation of the impact of initial saturation of an immiscible phase in the damaged zone on the efficiency of wormhole generation and growth in acidizing operations. We present a dimensionless two-phase reactive transport modelling tailored for studying the processes associated with rock acidizing. For a case study of acid injection into calcite with random porosity and permeability distribution, we show that an initial two-phase condition has positive feedback on the generation of wormholes. The results, however, indicate that the relative magnitude of reduction in the amount of pore volume of injected acid to produce effective wormholes depends on the mobility ratio, so that a higher mobility ratio facilitates a faster wormhole generation process. Under the conditions of modelling study presented, we demonstrate that in addition to the commonly used pair of Péclet-Damköhler regime identification, the mobility ratio of the displacing/displaced fluids, the relative permeability and phase condition need be accounted for in the analysis, if there are two-phase flow conditions across the target region of acidizing.
\end{abstract}

\section{Keywords}

matrix acidizing, two phase flow, reactive transport, wormholing process, rock heterogeneity

\section{1 - Introduction}

Reactions between acidic solutions and the solid phase appear in various engineering and natural subsurface processes including irrigating water discharge to aquifer systems (Valdes-Abellan et al., 2017), karst formation (Evans and Lizarralde, 2003; Zhao et al., 2013), contaminants transport in saturated and unsaturated soils (Cubillas et al., 2005; Chen et al., 2009; Nick et al., 2013; Atchley et al., 2014; Sedighi et al., 2015; Sedighi et al., 2016), radioactive waste remediation (Spycher et al., 2003), geological sequestration of carbon dioxide (e.g. Rochelle et al., 2004; Kampman et al., 2014; Islam et al., 2014; Islam et al., 2016), acid injection for enhanced oil recovery (e.g., Ghommem et al., 2015 and references therein) and acid-based enhanced geothermal systems (Portier et al., 2007;

\footnotetext{
${ }^{1}$ Corresponding author: Telephone +44 (0)161 306 4554, email: masoud.babaei@manchester.ac.uk
} 
Portier and Vuataz, 2010; Xiong et al., 2013; Na et al., 2016). To quantitatively predict the long-term consequences of subsurface reactions and their perturbations to the subsurface environment, reactive transport modelling serves as an instrumental and indispensable tool (Johnson et al., 2004). One of the challenging phenomena to predict in reactive transport modelling is the formation of preferential dissolution pathways in rock, also known as wormholes. These can occur as a result of feedback from geochemical interactions between rock and acidic solutions on fluid flow and chemical transport processes.

Over a span of decades, a compelling body of work has focused on developing transport modelling tools, validated by experiment, to predict the wormhole formation, propagation and stability in the context of oil recovery (Schechter and Gidley, 1969; Nierode et al., 1972; Daccord et al., 1989; Fredd and Fogler, 1998a; Golfier et al., 2006; Kalia and Balakotaiah, 2007; Cohen et al., 2008; Glasbergen et al., 2009; Qiu et al., 2013; Maheshwari et al., 2013; Ghommem et al., 2015;' Liu and Liu, 2016; Bastami and Pourafshary, 2016; Liu et al., 2017). In the context of petroleum engineering, acid solutions, typically 15 wt\% (Hung et al., 1989), are used to restore or enhance oil production that is affected by particles having migrated along the flow and having eventually accumulated near the wellbore region during the production time. In matrix acidizing, acidic solutions are injected at low pressures to dissolve preferably "some" of the rock and form highly conductive flow channels (wormholes). These preferential dissolution pathways are only generated if certain conditions of rock and injection parameters, including the rock's mineralogical configuration and distribution, and the rock's petrophysical properties and distribution are met with the right acid type, volume, dissolving power and injection rate (Davies and Kelkar, 2007). Therefore the interplay of flow and geochemistry determines the states at which wormholes are generated. The interplay is analysed through describing the parameters of flow and geochemistry via the Péclet number $(\mathrm{Pe})$ which is a dimensionless number describing the importance of advection in comparison with diffusion, and the Damköhler number $(D a)$, which is a dimensionless number equal to the ratio between the reaction rates and the dominant transport rate.

As shown in Figure 1, there are different dissolution regimes for different Da numbers, (i.e., different injection rates if the reaction rate is constant) as a result of the varying relative significance of the reaction with respect to the injection rate. At lower Da numbers (high injection rates), uniform dissolution patterns or ramified wormholes are generated, while at higher Da numbers, wormholegeneration dissolution or conical shaped dissolution patterns are generated. At even higher $D a$ numbers (not shown), the dissolution pattern is face dissolution, where the acid reacts soon after it contacts the medium. Therefore wormholing patterns are created near an intermediate or optimum value of the Damköhler number (in this example $D a=500$ ). In addition to requiring an optimal $D a-P e$ regime, the underlying physical and chemical conditions of rock can facilitate the desirable dissolution pattern of wormholes. For example, the mineralogical and petrophysical properties of carbonate rocks (limestone and dolomite) are favourable for the development of wormholes in certain Pe-Da regimes. Due to the existence of various length scales of pores in carbonates, when acid is injected in such media, the larger pores initially receive more acid and become enlarged due to the dissolution. This in turn means that acid is more likely to be transported into an enlarged pore causing even more dissolution. Therefore there is a positive feedback from geochemistry on the development and the extension of an eventual macroscopic channel. When fully developed, the wormholes naturally offer the least resistance path to the flow, as conductivity in these is orders of magnitude higher than in the unaltered or surrounding rock matrix (Liu et al., 2017). Thus the enhanced recovery can be achieved through the preferential pathways created (Lund et al., 1973). 


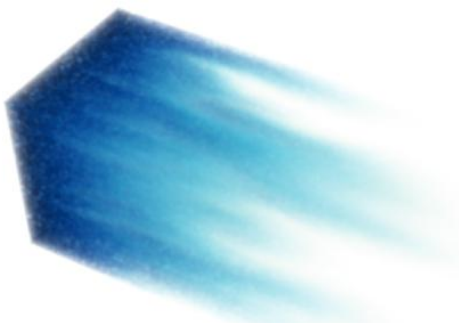

a) Uniform dissolution $(D a=1)$

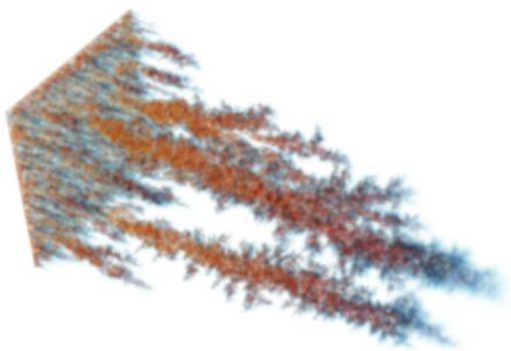

c) Wormhole dissolution $(D a=500)$

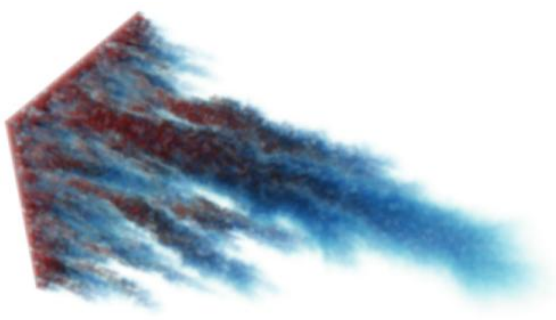

b) Ramified wormholes $(D a=40)$

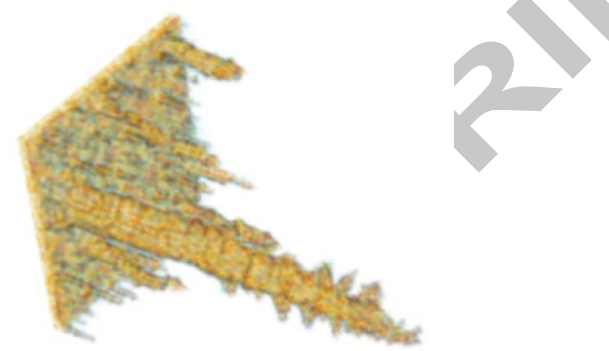

d) Conical dissolution $\left(D a=10^{4}\right)$

Abs. porosity increase

$\begin{array}{lllll}0.0 & 0.2 & 0.4 & 0.6 & 0.8\end{array}$

Figure 1 - Schematic diagram showing the transformation of the dissolution regime from uniform $(\mathrm{Da}=1)$ to wormholing $\left(\mathrm{Da}=10^{4}\right)$ in a $3 \mathrm{D}$ modelling example (for generating this figure. the code developed in this work has been applied for a single phase reactive transport problem, the specification of the porous media is the same as (Panga et al., 2005) extended to three dimensions).

In contrast to carbonates, siliciclastic (sandstone) acidizing (using mud acid, a mixture of $\mathrm{HF}$ and $\mathrm{HCl}$ ) is challenging due to the fact that there are multiple stages of fluid reaction with the minerals in the rock. While HF acid is used to dissolve the silicate minerals (clay, feldspar, and quartz), $\mathrm{HCl}$ enhances the dissolving power of $\mathrm{HF}$ with minerals by maintaining a low $\mathrm{pH}$ value through removal of carbonate minerals in the formation (Na et al., 2016). Nevertheless, unlike carbonates, the precipitation of fluorides, fluorosilicates, hydroxides, and amorphous silica caused by fluid-mineral interactions can potentially reduce the reservoir permeability (Shafiq and Mahmud, 2017). Finally, the generation of wormholes in fracture acidizing treatments (or acid fracturing) - where acid is used to keep the hydraulically generated fractures/cracks open by etching the faces of cracks after pressure is released-is deleterious (Hung et al., 1989) and should be avoided (Fredd, 2000) so that the acid solutions do not find their way out of the targeted zone and contaminate groundwater resources.

The importance of wormhole development can also be highlighted in the geological sequestration of carbon dioxide. The volume of $\mathrm{CO}_{2}$ injected into geological formations can be significantly greater than that of acid for well stimulation purposes - up to hundreds of cubic meters for acid stimulation vs. up to millions of cubic meters liquid $\mathrm{CO}_{2}$ for industrial-scale $\mathrm{CO}_{2}$ storage projects (Ott et al., 2013). Additionally the challenges to predict wormholing are more critical in the context of $\mathrm{CO}_{2}$ storage due to scale of the operation, uncertainty in $\mathrm{CO}_{2}$-fluid-rock interactions and multiphase conditions. While acid injection for enhanced oil production takes place in the wellbore region only (if contained successfully), $\mathrm{CO}_{2}$ fate and transport are determined across vast saline aquifers or depleted oil/gas reservoirs. The generation of wormholes in this context can jeopardise the safety and security of 
storage by generating highly conductive pathways of $\mathrm{CO}_{2}$ outside the targeted zone for sequestration.

The formation of wormholes is an important phenomenon with significant positive and negative implications. Its understanding and prediction are essential for economic viability, safety and success of geoengineering operations such as acid injection operations and $\mathrm{CO}_{2}$ storage. An area that has received limited attention on aspects of wormhole generation is the impact of phase saturation on the wormholing process. A $100 \%$ water-saturated or fully water-saturated (FWS) initial condition is the least likely initial condition when acid is injected into an oil-producing well, nevertheless it is the most common condition that has been used in laboratory studies of wormhole behaviour during carbonate acidizing (Shukla et al., 2006). Based on this assumption, the current numerical models of acid injection only consider single phase conditions (a fully water saturated wellbore), which in practice, near the wellbore, the porous media may contain pockets of immiscible oil or gas phases. As such, the modelling of reactive transport of acid under these conditions is worthy of scientific scrutiny. To the best knowledge of the authors, there is no published research focusing on "modelling" two-phase wormhole development. Against this knowledge gap, this paper aims to provide a better understanding of the effects of phase saturation on wormhole generation and development. Previously Shukla et al., (2006) and Kumar et al., (2014) have shown that the presence of an immiscible phase saturation prior to acid injection has significant impact on the efficacy of the operation. To provide a theoretical basis, a formulation and numerical model for matrix acidizing based on dimensional (Section 2) and dimensionless (Section 3) analyses for twophase injection of acid in reservoirs is presented. The model is specifically tailored to the calcite dissolution case in Section 4, and by performing flow-regime analyses with respect to phase saturation status, the results are compared with existing experimental findings on the impact of immiscible phase saturation on wormholing processes in Section 5.

\section{2 - Theoretical formulations}

A two-scale (pore scale and Darcy scale) approach is adopted and extended here for the reactive transport modelling of matrix acidizing in two-phase conditions. The model is based on an established approach of single phase advective-dispersive-reactive transport (Panga et al., 2005; Maheshwari and Balakotaiah, 2013; Liu et al., 2013; Ghommem et al., 2015). This approach has been shown to accurately capture the interplay of dissolution mechanisms that determines the critical rate (or Da-Pe values) at which the wormholes are generated across the wellbore, leading to an optimal enhancement of the permeability of the subsurface rock. The model incorporates the solidliquid interaction sequence: (1) diffusion of the liquid phase reactants to the immediate vicinity of the solid-liquid interface, (2) reaction at the solid-liquid interface between the concentrations of reactant-product at interface, (3) diffusion of the products away from the interface (Lund et al., 1973). We extend the single phase-based model to a two-phase flow formulation. The governing equation for phase flow, assuming that Darcy's law is applicable, may be written as:

$$
\frac{\partial \phi}{\partial t}+\nabla \cdot \mathbf{U}_{t}=0
$$

where $\phi$ represents the porosity and $\mathbf{U}_{t}$ is the total velocity of phases that is equal to $\left(\mathbf{U}_{w}+\mathbf{U}_{o}\right)$. $\mathbf{U}_{w}$ and $\mathbf{U}_{o}$ are velocity of water phase and oil phase, respectively.

The total velocity is calculated from: 


$$
\mathbf{U}_{t}=-\Lambda_{t} \mathrm{~K} \cdot \nabla P
$$

where $\Lambda_{t}$ is the total mobility, which is equal to sum of the mobility of water and oil phases (i.e. $\left.\Lambda_{w}+\Lambda_{o}\right)$. The mobility of each phase $(\alpha=w, o)$ is calculated by $\Lambda_{\alpha}=k_{r \alpha} / \mu_{\alpha}$, where $k_{r \alpha}$ and $\mu_{\alpha}$ are relative permeability and viscosity of phase $\alpha$, respectively. In this equation, $\mathrm{K}$ is the absolute permeability of the porous medium.

The mass conservation equation for the water phase flow can therefore be written as:

$$
\frac{\partial\left(\phi S_{w}\right)}{\partial t}+\mathbf{U}_{t} \cdot \nabla f_{w}=0
$$

where, $S_{w}$ is the degree of saturation of the water phase and $f_{w}=\Lambda_{w} / \Lambda_{t}$ is the fractional flow of the water phase. The reactive transport of acid is described based on the mass conservation law as:

$$
\frac{\partial\left(\phi S_{w} C_{f}\right)}{\partial t}+\mathbf{U}_{t} \cdot \nabla\left(f_{w} C_{f}\right)=\nabla \cdot\left(\phi S_{w} \mathbf{D}_{e} \nabla C_{f}\right)-a_{v} k_{c} S_{w}\left(C_{f}-C_{s}\right)
$$

where, $C_{f}$ is the concentration of acid in water phase and $\mathbf{D}_{e}$ is the effective dispersion tensor:

$$
\mathbf{D}_{e}=\left\{D_{m} \alpha_{0 s}+\xi_{X} P e_{L} D_{m}, \quad D_{m} \alpha_{0 s}+\xi_{T} P e_{T} D_{m}\right\}
$$

where $D_{m}$ is the molecular diffusion coefficient. The term $a_{v} k_{c} S_{w}$ in Eq.4 corresponds to the mass transfer of acid from the bulk fluid to the fluid-rock interface and includes the effects of mass loss of acid during dissolution of rock. In this term $a_{v}$ is the interfacial area available for reaction per unit volume of the medium. The solid dissolves at the fluid-solid interface. This gives rise to a concentration gradient. Since acid is depleted at the interface, the decrease in acid concentration at the interface is considered as a driver for mass transfer of acid from the bulk to the surface.

In Eq.5, $\alpha_{0 s} \xi_{X}$ and $\xi_{T}$ are coefficients of the dispersion tensor that depend on the structure of the porous medium (for example, tortuosity or connectivity between the pores and can be calculated from pore network modelling (Babaei and Joekar-Niasar, 2016). The coefficient of Péclet-number independent molecular diffusion in diffusion dominant regimes is $\alpha_{0 s}$, whereas $\xi_{X}$ and $\xi_{T}$ are the coefficients of longitudinal and transverse hydraulic dispersion in advection-controlled regimes.

The mass transfer acid from the bulk to the surface is related to the reaction of acid at rock surface. This enables us to calculate the temporal rate of acid concentration at the interface from the concentration of the acid in the bulk fluid. Therefore, the rate of mass transfer from the bulk fluid to the interface $\left(R_{t}=\partial C_{f} / \partial t\right)$ can be calculated as:

$$
R_{t}=k_{c}\left(C_{f}-C_{s}\right)
$$

where, $k_{c}$ is the local mass-transfer coefficient and $C_{s}$ is the concentration of acid at the solid-fluid interface. 
The chemical reaction rate of acid at the solid liquid interface $\left(R_{s}=\partial C_{s} / \partial t\right)$ can be assumed to be a first order kinetic reaction -with reaction rate constant of $k_{s}$ - given as (Steefel and Lasaga, 1994; Fu et al., 2015):

$$
R_{s}=k_{s} f\left(C_{s}\right)=k_{s} C_{s}
$$

At stationary state, the rate of reactant diffusional transport towards the surface is equal to the rate at which these reactants are consumed at the solid surface (Figure 2), i.e., $R_{s}=R_{t}$. It is therefore possible to calculate the rate of chemical changes at the solid-fluid interface as a function of acid concentration in bulk fluid:

$$
\frac{\partial C_{s}}{\partial t}=\frac{k_{c} k_{s}}{k_{c}+k_{s}} C_{f}
$$

where $k_{c} k_{s} /\left(k_{c}+k_{s}\right)$ is the overall or observed rate constant and is analogous to the rate of solidcatalysed reaction (Davis and Davis, 2012). Finally, the feedback of acid reaction to the pore space (porosity development due to reaction) is modelled by:

$$
\frac{\partial\left(\phi S_{w}\right)}{\partial t}=\frac{k_{c}\left(C_{f}-C_{s}\right) a_{v} \alpha}{\rho_{s}}
$$

where $\alpha$ is the dissolving power of acid (grams of solid dissolved per mole of acid reacted) and $\rho_{s}$ is the density of solid phase.

The variables in the Darcy scale model described above are the permeability tensor, the dispersion tensor, the mass transfer coefficient and the interfacial area available for reaction per unit volume of the medium. These variables are dependent on the pore structure. Structure-property relations are used, which relate permeability, interfacial surface area available for reaction and average pore radius to porosity (Wyllie and Gardner, 1958):

$$
\begin{gathered}
\frac{\mathrm{K}}{\mathrm{K}_{0}}=\frac{\phi}{\phi_{0}}\left(\frac{\phi\left(1-\phi_{0}\right)}{\phi_{0}(1-\phi)}\right)^{2 \beta} \\
\frac{r_{p}}{r_{0}}=\sqrt{\frac{\mathrm{K} \phi_{0}}{\mathrm{~K}_{0} \phi}} \\
\frac{a_{v}}{a_{0}}=\frac{\phi r_{0}}{\phi_{0} r_{p}}
\end{gathered}
$$

where $\mathrm{K}_{0}, r_{0}$ and $a_{0}$ are the initial values of permeability, average pore radius and interfacial area, respectively, $\beta$ is the pore broadening parameter, and $\mathrm{K}, r_{p}$ and $a_{v}$ are the dynamically varying permeability, average pore radius and interfacial area, respectively. 


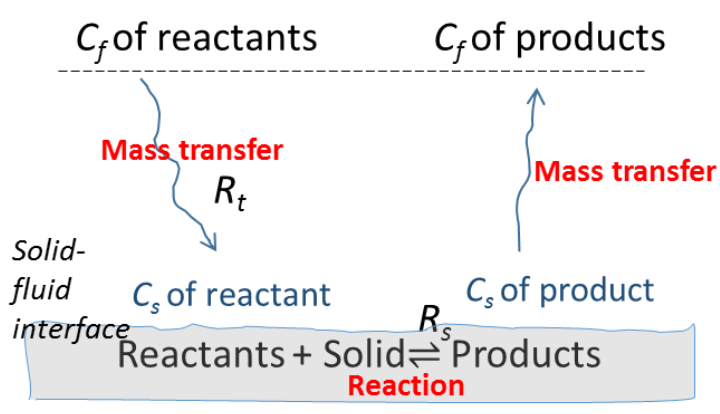

Boundary layer

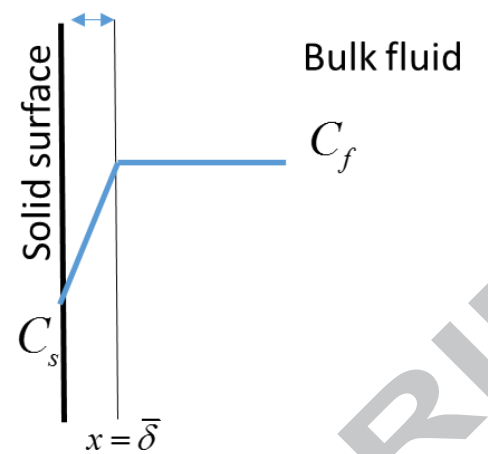

Figure 2 - The conceptualization of the fluid-solid interaction in the model.

The model is capable to capture both the extremes of solid-fluid interaction regimes from kineticallycontrolled, where the reaction rate between the solid and fluid is slow, to mass transfer (or diffusion) controlled, where the reaction takes place very fast at the solid-fluid interface, and the kinetics of the reaction can be limited either by reactant transport towards the solid-liquid interface or transport of the products away from the interface (Daccord et al., 1993). Nevertheless, we clarify that the number of physical phenomena that occurs between the phases and solid (such as wetting condition, contact angle and capillarity that govern the initial distribution, mixing and mobilisation of wetting/non-wetting immobile phase within a non-wetting/wetting phase) are neglected. These phenomena can affect the mass transfer and surface reaction rates. Against this backdrop, the formulation presented in this work (over) simplifies the pore scale physics. More rigorous physicsbased pore-scale models that can capture coupled non-linear multiphase flow with phase separation, mass transport, chemical reaction, dissolution-precipitation, and dynamic evolution of the pore geometries at pore scale (e.g., Parmigiani et al., 2011; Chen et al., 2013; Liu and Mostaghimi, 2017a; Liu and Mostaghimi, 2017b) can be coupled with the continuum scale model developed here .Additionally, the two-phase formulation presented here requires a new set of twophase experimentations for specific wetting conditions of the rock (water wet vs. oil wet vs. mixed wet) and observed initial distribution of the immiscible phase in the core. Unfortunately the experiments of Shukla et al. (2006) did not report this information.

\section{3 - Dimensionless form of the governing equations}

A dimensionless form of the governing equations is developed here that facilitates a comparative setting for obtaining the ideal $P e$ - $D a$ regime at which effective wormholing can be achieved. The dimensionless form also facilitates the condensing of a number of variables to a few dimensionless key parameters such as $D a, P e$ and mobility ratio $(M)$. In order to develop the dimensionless theoretical equations presented in the previous section, we define a series of dimensionless variables as reported in Table 1.

Table 1 -Dimensionless variables used to non-dimensionalize the Darcy-scale two-phase model

\begin{tabular}{ll}
\hline Variable & Description \\
\hline$x^{*}=x / L, y^{*}=y / L$ & Dimensionless position with respect to the characteristic length $L$ \\
$t^{*}=t /\left(L / u_{0}\right)$ & Dimensionless time with respect to the inlet velocity, $u_{0}$ \\
$\mathbf{u}_{t}=\mathbf{U}_{t} / u_{0}$ & Dimensionless total Darcy flux \\
\hline
\end{tabular}




\begin{tabular}{|c|c|}
\hline$r=r_{p} / r_{0}$ & Dimensionless average pore radius \\
\hline$A_{v}=a_{v} / a_{0}$ & Dimensionless interfacial area, \\
\hline$\kappa=\mathrm{K} / \mathrm{K}_{0}$ & Dimensionless permeability \\
\hline$c_{f}=C_{f} / C_{0}, c_{s}=C_{s} / C_{0}$ & $\begin{array}{l}\text { Dimensionless concentrations with respect to the inlet concentration } \\
\text { of the acid, } C_{0}\end{array}$ \\
\hline$p=\left(P-P_{\text {out }}\right) /\left(u_{0} L / \Lambda_{0} \mathrm{~K}_{0}\right)$ & $\begin{array}{l}\text { Dimensionless pressure with respect to the constant pressure at } \\
\text { boundary, } P_{\text {out }} \text {, and the total mobility } \Lambda_{0}=\Lambda_{t} \text { at } S_{w}=1.0 \\
\text { (single phase water) }\end{array}$ \\
\hline$\lambda=\Lambda_{t} / \Lambda_{0}$ & $\begin{array}{l}\text { Dimensionless total mobility with respect to the total mobility at } \\
\qquad S_{w}=1.0\end{array}$ \\
\hline$h_{T}^{2}=2 k_{s} r_{0} / D_{m}$ & $\begin{array}{l}\text { Pore scale Thiele modulus (reaction rate over diffusion rate) with } \\
\text { respect to molecular diffusion } D_{m}\end{array}$ \\
\hline$H_{T}^{2}=k_{s} a_{0} L^{2} / D_{m}=D a P e_{L}$ & Macroscopic Thiele modulus \\
\hline$D a=k_{s} a_{0} L / u_{0}$ & Damköhler number \\
\hline$N_{a c}=\alpha C_{0} / \rho_{s}$ & Acid capacity number \\
\hline$P e_{L, T}=u_{0} L / D_{m}$ & Longitudinal $(L)$ or transversal $(T)$ Péclet number \\
\hline$\eta=2 r_{0} / L$ & Ratio of average pore diameter to characteristic length \\
\hline$S h=2 k_{c} r_{p} / D_{m}$ & $\begin{array}{l}\text { Sherwood number (the ratio of the convective mass transfer to the } \\
\text { rate of diffusive mass transport) }\end{array}$ \\
\hline
\end{tabular}

By replacing the dimensionless variables of Table 1 in formulations for pressure (Eq.1 and Eq.2), saturation (Eq.3), concentration (Eq.4), porosity development (Eq.9) and the empirical relations (Eq.10, Eq.11 and Eq.12) the following dimensionless formulations are obtained.

The phase flow equation can be simplified to:

$$
\begin{gathered}
\mathbf{u}_{t} u_{0}=-\Lambda_{t} \mathrm{~K} \frac{\partial}{\partial x^{*} L}\left(\frac{u_{0} L}{\Lambda_{0} \mathrm{~K}_{0}} p\right) \rightarrow \mathbf{u}_{t}=-\lambda \kappa \nabla^{*} p \\
\frac{\partial \phi}{\partial t^{*} L / u_{0}}+\frac{\partial u_{0} \mathbf{u}_{t}}{\partial x^{*} L}=0 \rightarrow \frac{\partial \phi}{\partial t^{*}}+\nabla^{*} \cdot \mathbf{u}_{t}=0
\end{gathered}
$$

Eq.14 states that the porosity change will impact the dimensionless velocity. The saturation and concentration equations are changed to:

$$
\begin{gathered}
\frac{\partial\left(\phi S_{w}\right)}{\partial t^{*} L / u_{0}}+u_{0} \mathbf{u}_{t} \frac{\partial f_{w}}{\partial x^{*} L}=0 \rightarrow \frac{\partial\left(\phi S_{w}\right)}{\partial t^{*}}+\mathbf{u}_{t} \cdot \nabla^{*} f_{w}=0 \\
\frac{\partial\left(\phi S_{w} c_{f} C_{0}\right)}{\partial t^{*} L / u_{0}}+\frac{\partial\left(\mathbf{u} u_{0} c_{f} C_{0}\right)}{\partial x^{*} L}=\frac{\partial}{\partial x^{*} L}\left(S_{w} \phi\left(D_{m} \alpha_{0 s}+\xi_{X} P e_{L} D_{m}\right) \frac{\partial c_{f} C_{0}}{\partial x^{*} L}\right) \\
+\frac{\partial}{\partial y^{*} L}\left(S_{w} \phi\left(D_{m} \alpha_{0 s}+\xi_{T} P e_{T} D_{m}\right) \frac{\partial c_{f} C_{0}}{\partial y^{*} L}\right) \\
-A_{v} a_{0} k_{c} S_{w}\left(c_{f} C_{0}-c_{s} C_{0}\right)
\end{gathered}
$$




$$
\begin{aligned}
\frac{\partial\left(\phi S_{w} c_{f}\right)}{\partial t^{*}}+\frac{\mathbf{u}_{t} \partial\left(S_{w} c_{f}\right)}{\partial x^{*}}=\frac{\partial}{\partial x^{*}} & \left(S_{w}\left(\phi \frac{D a}{H_{T}^{2}} \alpha_{0 s}+\xi_{X}\left|\mathbf{u}_{t}\right| \eta r\right) \frac{\partial c_{f}}{\partial x^{*}}\right) \\
& +\frac{\partial}{\partial y^{*}}\left(S_{w}\left(\phi \frac{D a}{H_{T}^{2}} \alpha_{0 s}+\xi_{T}\left|\mathbf{u}_{t}\right| \eta r\right) \frac{\partial c_{f}}{\partial y^{*}}\right)-\frac{A_{v} S_{w} c_{f} D a}{1+\frac{h_{T}^{2} r}{S h}}
\end{aligned}
$$

Here we have purposely used $y$-direction denotations as well to show where the transverse dispersion coefficient is used. The porosity development equation is changed to:

$$
\frac{\partial\left(\phi S_{w}\right)}{\partial t}=\frac{A_{v} N_{a c} S_{w} c_{f} D a}{1+\frac{h_{T}^{2} r}{S h}}
$$

The empirical equations are non-dimensionalized as:

$$
\begin{gathered}
\kappa=\frac{\phi}{\phi_{0}}\left(\frac{\phi\left(1-\phi_{0}\right)}{\phi_{0}(1-\phi)}\right)^{2 \beta} \\
r=\sqrt{\frac{\kappa \phi_{0}}{\phi}} \\
A_{v}=\frac{\phi}{\phi_{0} r}
\end{gathered}
$$

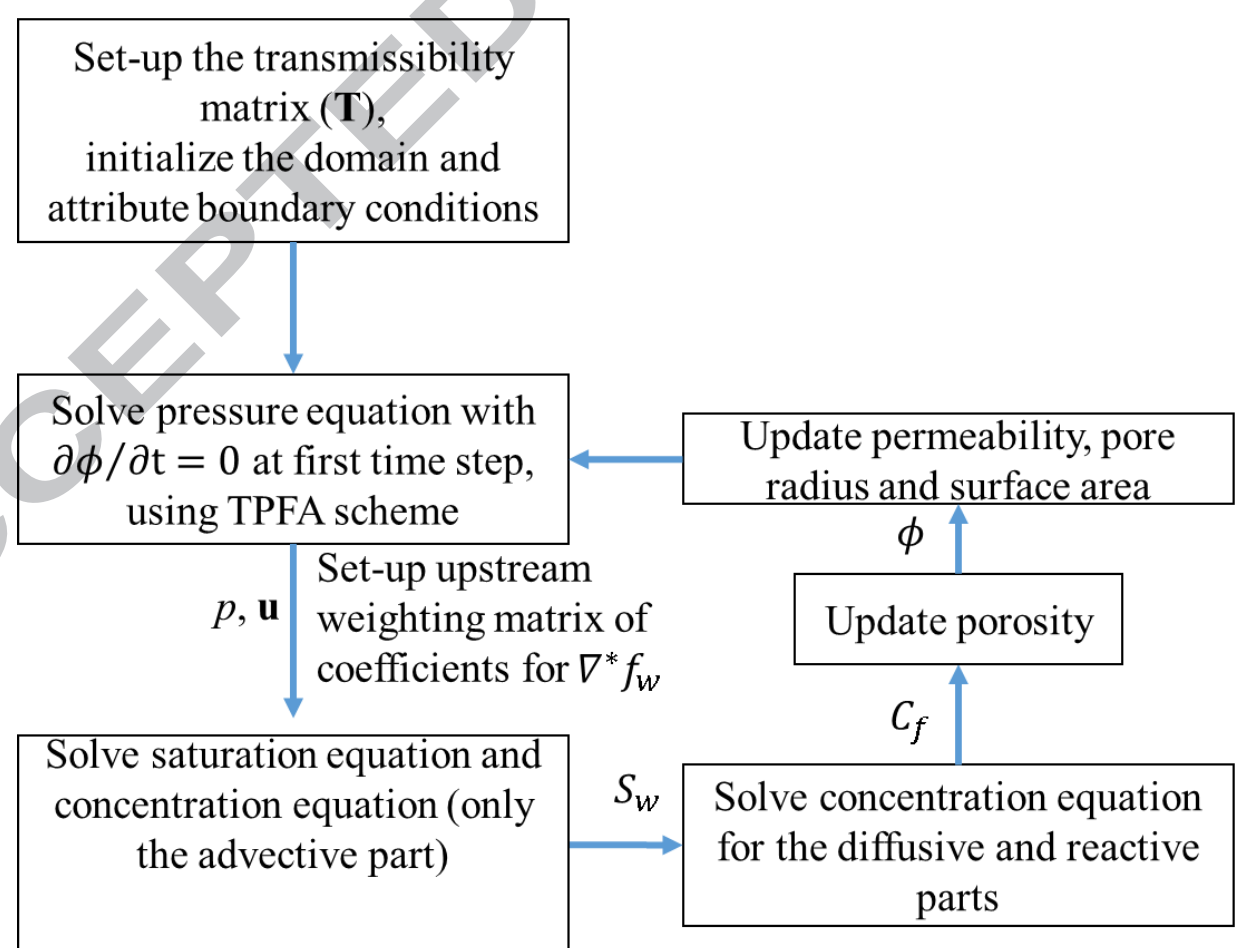

Figure 3 - The algorithm of solving two-phase acid injection.

We solve this equation using a finite difference two-point-flux-approximation scheme (TPFA) described in (Babaei and King, 2012). The base code for water-oil displacement with solute concentration in injection fluid has been tested previously (Babaei and King, 2013). Having solved 
for the dimensionless pressure, the dimensionless flux is extracted from the pressure solution via Eq.13. Next, the dimensionless saturation equation (Eq.15) is solved explicitly using an upstream weighting scheme for flux across the interfaces. Finally, the dimensionless concentration equation (Eq.16) is solved using operator splitting for advection, diffusion and reaction.

We use the Courant-Friedrichs-Lewy condition to break the time steps for saturation and concentration. After each time step, first the porosity development is calculated via Eq.17, and then the dimensionless permeability, the dimensionless average pore radius and the dimensionless interfacial area are updated using Eq.18, Eq.19 and Eq.20, respectively. The algorithm as such is sequential and for single phase condition has been validated against experimental results of acid injection by recent publications (Maheshwari et al., 2013; Ghommem et al., 2015).

\section{4 - Calcite dissolution with $\mathrm{HCl}$ acid}

Wormhole development during matrix acidizing of partially saturated calcite is studied here to investigate the impact of an existing immiscible phase distributed homogeneously across the domain. The aim is to investigate the impact of mobility ratio (the mobility of displacing fluid over the mobility of displaced fluid) on the optimal conditions of acid injection (Pe-Da configurations) at which the wormholes are generated with minimum amount of acid injected. We hereafter denote this optimal amount of acid as $\mathrm{PV}_{\mathrm{BT}}$. The problem considered is a synthetic core sample of calcite. The dissolution of calcite can be presented as Fredd and Fogler, (1998b) and Morse and Arvidson, (2002):

$$
\mathrm{CaCO}_{3}(\mathrm{~s})+\mathrm{H}^{+} \leftrightarrow \mathrm{Ca}^{2+}+\mathrm{HCO}_{3}^{-}
$$

The chemical reaction rate is given by Alkattan et al., (2002) and Morse and Arvidson, (2002):

$$
R_{s}=k_{s}^{\prime}\left(a_{\mathrm{H}^{+}, \mathrm{s}}\right)^{n}=k_{s}^{\prime}\left(\gamma_{\mathrm{H}^{+}, \mathrm{s}} C_{\mathrm{H}^{+}, \mathrm{s}}\right)^{n}
$$

where $a_{\mathrm{H}^{+}, \mathrm{s}}, \gamma_{\mathrm{H}^{+}, \mathrm{s}}$ and $C_{\mathrm{H}^{+}, \mathrm{s}}$ represent the activity, activity coefficient, and concentration of aqueous $\mathrm{H}^{+}$adjacent to the calcite surface. Based on the experimental measurements of Plummer et al., (1978) and Compton et al., (1989), we assume $n=1$. Protons consumed by Eq.21 leads to diffusion concentration gradients near the calcite surface that can be described using:

$$
R_{t}=k_{c}\left(C_{\mathrm{H}^{+}, \mathrm{b}}-C_{\mathrm{H}^{+}, \mathrm{s}}\right)
$$

where $R_{t}$ is, as defined in Eq. 6 for the general case, the transport rate by mass transfer (diffusion) from bulk to surface-fluid interface (equivalent to $C_{f}$ to $C_{s}$ ). Similarly these rates are equal to rate of products diffusional transport away from the surface. By combining Eq.21 and Eq.22, and analogous to Eq.8:

$$
C_{\mathrm{H}^{+}, \mathrm{s}}=\frac{k_{c} C_{\mathrm{H}^{+}, \mathrm{b}}}{k_{s}^{\prime} \gamma_{\mathrm{H}^{+}, \mathrm{s}}+k_{c}}
$$

and; 


$$
R_{s}=\frac{k_{c} k_{s}^{\prime} \gamma_{\mathrm{H}^{+}, \mathrm{s}}}{k_{s}^{\prime} \gamma_{\mathrm{H}^{+}, \mathrm{s}}+k_{c}} C_{\mathrm{H}^{+}, \mathrm{b}}=\frac{k_{c} k_{s}}{k_{s}+k_{c}} C_{f},
$$

where $k_{c} k_{s} /\left(k_{s}+k_{c}\right)$ is the overall (observed) calcite dissolution rate constant that is a function of

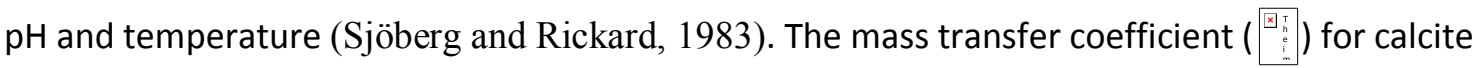
dissolution, depends strongly on the geometry (Golfier et al., 2001), and can be calculated by volume averaging from pore scale modelling as a function of Péclet number and macroscopic porosity (Golfier et al., 2006, Fig. 10). Despite the variations in the mass transfer coefficient, we use

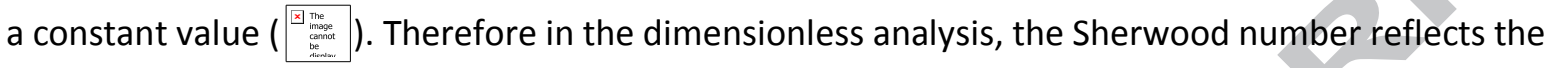
value for the mass transfer coefficient. Similarly the Damköhler number is a reflection of $k_{s}$.

In our study we ignore the impact of products of calcite/dolomite dissolution on the overall performance of acidizing operation. The produced insoluble $\mathrm{CO}_{2}$ may change the relative permeability of the reactants and influence on the optimum conditions (Bastami and Pourafshary, 2016), if a separate $\mathrm{CO}_{2}$ phase is formed.

We assume a domain in 2D. We use a uniform discretisation of 250 by 100 mesh corresponding to $0.2 \mathrm{~cm} \times 0.2 \mathrm{~cm}$ gridblocks. The variables required for modelling the case study are listed in Table 2 . With regards to the flow boundary conditions of the problem, the fluid injection takes place at the left hand side of the domain at a constant injection rate (i.e., constant flux that varies in the different scenarios studied). The flow boundary condition at right hand side of the core is set to be a constant pressure of $1 \mathrm{~atm}$ throughout the simulation. The concentration of acid in the fluid at the right hand side of the domain is set to be equal to the initial value.

Table 2 - The properties of fluid and rock used unchanged between all the simulation cases in this

\begin{tabular}{cccc}
\multicolumn{4}{c}{ study. } \\
\hline Variables & Values assigned & Variables & Values assigned \\
\hline$L_{x}$ & $50 \mathrm{~cm}$ & $S h$ & 2 \\
$L_{y}$ & $20 \mathrm{~cm}$ & $D_{m}$ & $10^{-9} \mathrm{~m}^{2} / \mathrm{sec}$ \\
$r_{0}$ & $0.5 \times 10^{-6} \mathrm{~m}$ & $h_{T}^{2}$ & 0.07 \\
$\alpha_{0 s}$ & 0.4 & $H_{T}^{2}$ & $10^{6}$ \\
$\xi_{X}$ & 0.5 & $N_{a c}$ & 0.1 \\
$\xi_{T}$ & 0.1 & $P_{\text {out }}$ & $1 \mathrm{~atm}$ \\
$C_{0}$ & 0 & $\beta$ & 2 \\
\hline
\end{tabular}

In summary, the boundary conditions applied can be summarised as:

$$
\begin{gathered}
U=-\kappa \lambda \frac{\partial p}{\partial x^{*}}=u_{0}, \quad \lambda=\Lambda_{w} / \Lambda_{w}=1 \quad \text { at } x^{*}=0 \\
p=0 \quad \text { at } x^{*}=L \\
C_{f}=C_{0} \quad \text { at } x^{*}=L
\end{gathered}
$$

Boundary conditions at the top and base of the sample are set to be no-flow (i.e., $\partial p / \partial y^{*}=0$ ). 
A great variety of phenomenological models have been suggested to interrelate the dimensionless modified Sherwood number with Reynolds, and Schmidt numbers. For example $S h=S h_{\infty}+\beta_{0} \operatorname{Re}^{\beta_{1}}\left(S_{\alpha} / \phi\right)^{\beta_{2}} S c^{\beta_{3}}$ with $\beta_{0}, \beta_{1}, \beta_{2}$ and $\beta_{3}$ as fitting parameters (Qin et al., 2007). In this empirical relationship, the diffusion-induced mass transfer is given by $S h_{\infty}$, and the convectioninduced mass transfer is $\beta_{0} R e^{\beta_{1}}\left(S_{\alpha} / \phi\right)^{\beta_{2}} S c^{\beta_{3}}$. In our study we assume $S h=2+0.6 R e^{1 / 2} S c^{1 / 3}$, that corresponds to flow in a packed bed of spheres (Panga et al., 2005). This assumption can be relaxed if information about pore morphology is available. The effect of reaction rate on the mass transfer coefficient has been observed to be weak (Balakotaiah and West, 2002), and the effect of the convective mass transfer on acid consumption may not be significant because of the extremely low interfacial area in the high porosity regions where convection is dominant (Panga et al., 2005). Therefore we assume $S h=2$.

We assume Corey correlations of the relative permeability of oil and water, as in:

$$
\begin{gathered}
k_{r o}\left(S_{w}\right)=k_{r o}^{0}\left(1-S_{w n}\right)^{n_{o}}, \\
k_{r w}\left(S_{w}\right)=k_{r w}^{0}\left(S_{w n}\right)^{n_{w}} \\
S_{w n}=\frac{S_{w}-S_{w c}}{1-S_{w c}-S_{o r}}
\end{gathered}
$$

where $k_{r o}^{0}$ and $k_{r w}^{0}$ are the end-point relative permeability multipliers, and $S_{w c}$ is the connate water saturation and $S_{o r}$ is the residual oil saturation. For the relative permeability relationships, we assume $n_{w}=n_{o}=2, S_{w c}=S_{o r}=0, k_{r w}^{\max }=k_{r o}^{\max }=1.0$ in all cases. Using the above relative permeability relationships, for fractional flow of water, it can be written:

$$
f_{w}\left(S_{w}\right)=\frac{1}{1+\frac{k_{r o} \mu_{w}}{k_{r w} \mu_{o}}}=\frac{1}{1+\frac{k_{r o}^{\max } \mu_{w}\left(1-S_{w n}\right)^{n_{o}}}{k_{r w}^{\max } \mu_{o}\left(S_{w n}\right)^{n_{w}}}}=\frac{1}{1+\frac{1}{M} \frac{\left(1-S_{w n}\right)^{n_{o}}}{\left(S_{w n}\right)^{n_{w}}}}
$$

where, $M$ is the mobility ratio (mobility of injection water divided by displaced oil).

In order to define rigorous criteria for wormhole generation detection we use the ratio of pressure difference at any simulation time $t$ over initial pressure $\left(\Delta p_{t} / \Delta p_{0}\right)$ as a measure of how effective wormholes could reduce the pressure difference. If the dimensionless pressure $\operatorname{drop}\left(\Delta p_{t} / \Delta p_{0}\right)$ has become less than or equal to 0.01 , we assume the wormholes are generated and have fully grown to create superconductive flow paths so that the pressure difference has dropped to almost zero across the domain. This criterion for $\mathrm{PV}_{\mathrm{BT}}$ has been used by (Kalia and Balakotaiah, 2007) as opposed to the criteria based on the breakthrough $\left(C_{f}>\varepsilon\right)$. The criterion based on $\Delta p_{t} / \Delta p_{0}$ offers a less subjective and more practical approach in determining $\mathrm{PV}_{\mathrm{BT}}$.

To delineate the impact of phase saturation, we define a number of simulation cases with various

\begin{tabular}{|c|c|c|c|c|}
\hline Simulations & Initial phase & Mobility ratio & Péclet number & Damköhler \\
\hline
\end{tabular}
initial water saturations, Pe numbers (injecting with different $u_{0}$ ) and $D a$ numbers (assuming different $k_{s}$ values). The simulation cases are defined in Table 3.

Table 3 - Definition of the simulation cases. 


\begin{tabular}{|c|c|c|c|c|}
\hline suite no. & saturation & $M=\frac{k_{r w}^{\max } \mu_{o}}{k_{r o}^{\max } \mu_{w}}$ & $\left(Q_{0} L_{x}\right) /\left(D_{m} L_{y} L_{z}\right)$ & numbers \\
\hline 1 & $\begin{array}{c}1.0\left(\mathrm{FWS}^{2}\right) \text { and } \\
0.5\end{array}$ & $\frac{1 \times 10 \mathrm{mPa} \cdot \mathrm{sec}}{1 \times 1 \mathrm{mPa} \cdot \mathrm{sec}}=10$ & $2.5 \times 10^{3}$ & $\begin{array}{c}0.1,1,10,100 \\
500,1000\end{array}$ \\
\hline 2 & 1.0 and 0.5 & $\frac{1 \times 10 \mathrm{mPa} \cdot \mathrm{sec}}{1 \times 1 \mathrm{mPa} \cdot \mathrm{sec}}=10$ & $2.5 \times 10^{5}$ & $\begin{array}{c}0.1,1,10,100 \\
500,1000\end{array}$ \\
\hline 3 & 1.0 and 0.5 & $\frac{1 \times 1 \mathrm{mPa} \cdot \mathrm{sec}}{1 \times 1 \mathrm{mPa} \cdot \mathrm{sec}}=1$ & $2.5 \times 10^{5}$ & $\begin{array}{l}0.1,1,10,100 \\
500,1000\end{array}$ \\
\hline 4 & 1.0 and 0.5 & $\frac{1 \times 1 \mathrm{mPa} \cdot \mathrm{sec}}{1 \times 10 \mathrm{mPa} \cdot \mathrm{sec}}=0.1$ & $2.5 \times 10^{5}$ & $\begin{array}{l}10,100 \\
1000\end{array}$ \\
\hline
\end{tabular}

For the initial permeability $\left(\mathrm{K}_{0}\right)$ we use a random distribution between $1 \mathrm{mD}$ to $1000 \mathrm{mD}$ (without spatial correlation). The initial porosity $\left(\phi_{0}\right)$ is also randomly distributed with the values of $0.2 \pm 0.15$ (random distribution between 0.05 and 0.35 with mean of 0.2 ). It is noted that the porosity is uncorrelated with permeability. To generate random distribution we use a MATLAB utility function rand. Further realistic distribution of porosity and permeability can be considered in the simulation by performing core petrophysical analysis. For the current work, we use the most simplistic porositypermeability distribution.

\section{5 - Results and discussion}

We start our analysis with simulation suite 1 and report the results by plotting $\Delta p_{t} / \Delta p_{0}$ vs. initial pore volume injected (PVI) (the pore volume before acid injection). As shown in Figure 4, we observe that a faster rate of decrease in $\Delta p_{t} / \Delta p_{0}$ is observed for $S_{w}=0.5$ for all the Da numbers which shows the wormholes have been generated more effectively across the domain for $S_{w}=0.5$ than FWS. It can be observed that there is an optimal $D a$ number for $P V_{B T}$ when $P e=2.5 \times 10^{3}$, this value is around $D a=1$ to 10 for both FWS and $S_{w}=0.5$ simulations.

The impact of the initially unsaturated domain on pressure reduction is significant for suboptimal $D a$ regimes (smaller or larger than the optimal value). At the optimal $D a$ regime of $D a=10$, there is approximately 1.0 PVI (from 3.8 to 2.84) less required amount of acid to have $\Delta p_{t} / \Delta p_{0}=0.01$ by $S_{w}$ $=0.5$ than FWS. This decrease corresponds to approximately a $25 \%$ reduction in the PVI required for breakthrough. These results have been observed experimentally by (Shukla et al., 2006), for the case of the presence of an immiscible gas saturation (nitrogen-saturated with residual water saturation). The presence of an immiscible phase can reduce the fluid loss from the main wormhole, thus allowing for deeper penetration of wormholes with a given acid volume (Shukla et al., 2006).

\footnotetext{
${ }^{2}$ Fully water saturated
} 

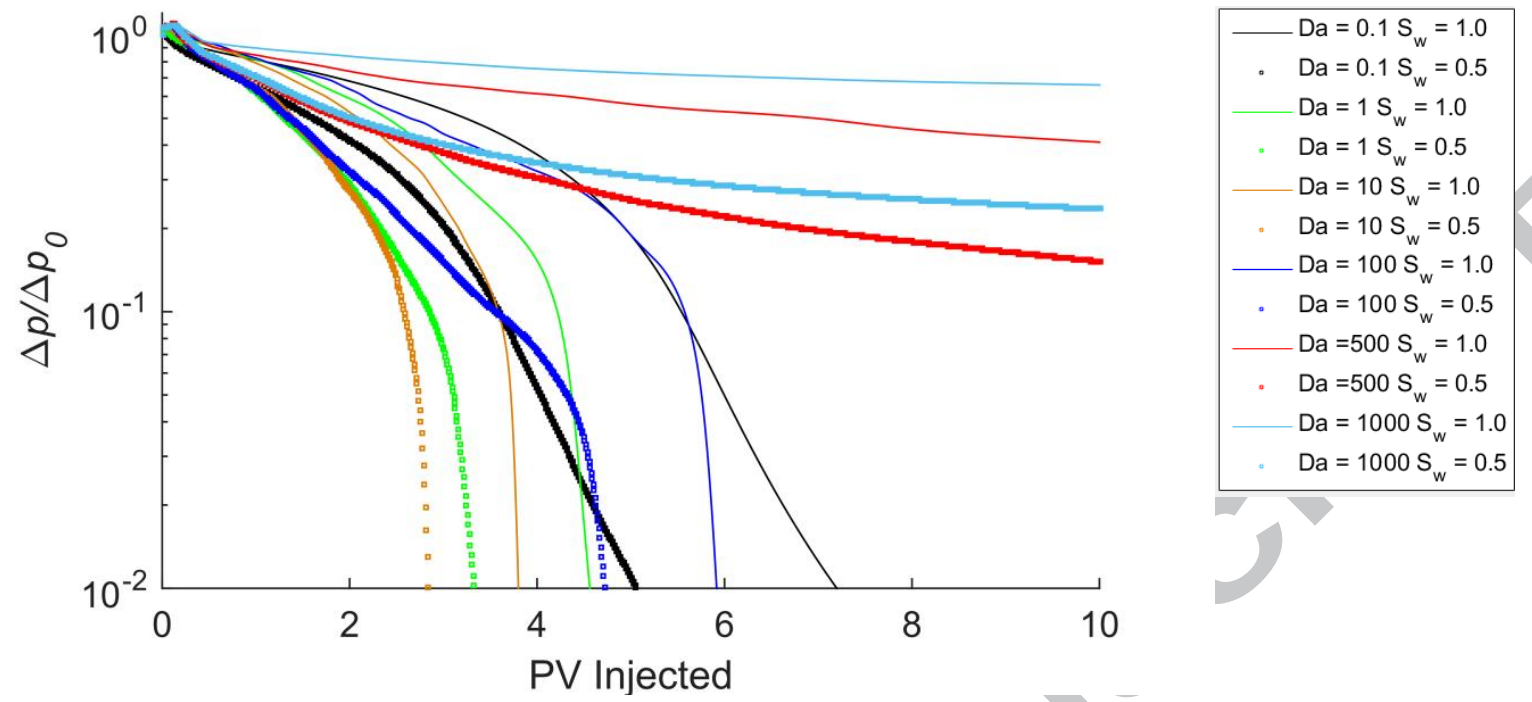

Figure 4 - The pressure gradient reduction vs. initial pore volume injection for suite 1 of simulations.

The oil present $\left(S_{o}=0.5\right)$ in two-phase conditions contributes towards the fewest pore volumes of acid being used for reducing the pressure gradient across the domain by acting as a buffer zone between the rock and acid. The acid flows through the wormholes and is not spent dissolving the rock matrix. For fully water saturated cases, the relative permeability of acid through the domain is unchanged, resulting in greater fluid loss to the regions surrounding the main wormhole.

Consequentially, higher pore volumes of acid are consumed for creating a wormhole. The authors also hypothesize that the underlying fundamental physical factor that enhances the wormhole faster development and growth in two-phase conditions than the fully-saturated condition is the appearance of dead-end pore volume under the two phase conditions (De Gennes, 1983; De Smedt and Wierenga, 1984). Such dead-end spaces (immobile zones) interact with the main "backbone/mobile zone" part only through rate-limited molecular diffusion. A compelling volume of scientific works have focused on the mobile/immobile zonation in hydrodynamics. Recently Karadimitriou et al. (2016) have also visualized this partitioning in two-phase micromodel experiments. The appearance of hard to access immobile zones under two-phase conditions means that the solute (acid) can only access mobile zones. This has a direct impact on wormhole generation as the reaction is automatically taking place in more limited areas. Further investigation of the process requires an in-depth analysis of the physics at pore-scale level under the reactive-advectivedispersive "two-phase" condition.

In Figure 5 we presented a $90^{\circ}$ flipped profiles of saturation, concentration and porosity increase ( $\phi-\phi_{0}$ ) for simulation cases in suite. The simulations for $D a$ up to 5000 have been unable to generate conical and face dissolution regimes. These regimes are generated at high Da numbers (Panga et al., 2005) when fluid dispersion and any perturbations in the reaction front are suppressed by transverse dispersion (Kalia and Balakotaiah, 2009).

The results are not shown here, however, we have simulated the cases with $D a=10,000$ and $D a=$ 30,000 with 2 orders of magnitudes smaller dimensionless time-steps (because of the strong reactions) than what is used for the simulation results of this study $\left(d t^{*}=0.01\right)$ and both conical and face dissolution regimes were recorded. These regimes however are suboptimal for wormholing and do not change the conclusions regarding the impact of unsaturated porous media on the wormholing process. 
The speed of wormholes propagation is enhanced by presence of an immiscible phase, so that, for example, for $D a=100$, it takes $4.72 \mathrm{PVI}$ for $S_{w}=0.5$ to reach $\Delta p_{t} / \Delta p_{0}=0.01$, while it takes $5.91 \mathrm{PVI}$ for $S_{w}=1.0$ to reach $\Delta p_{t} / \Delta p_{0}=0.01$.

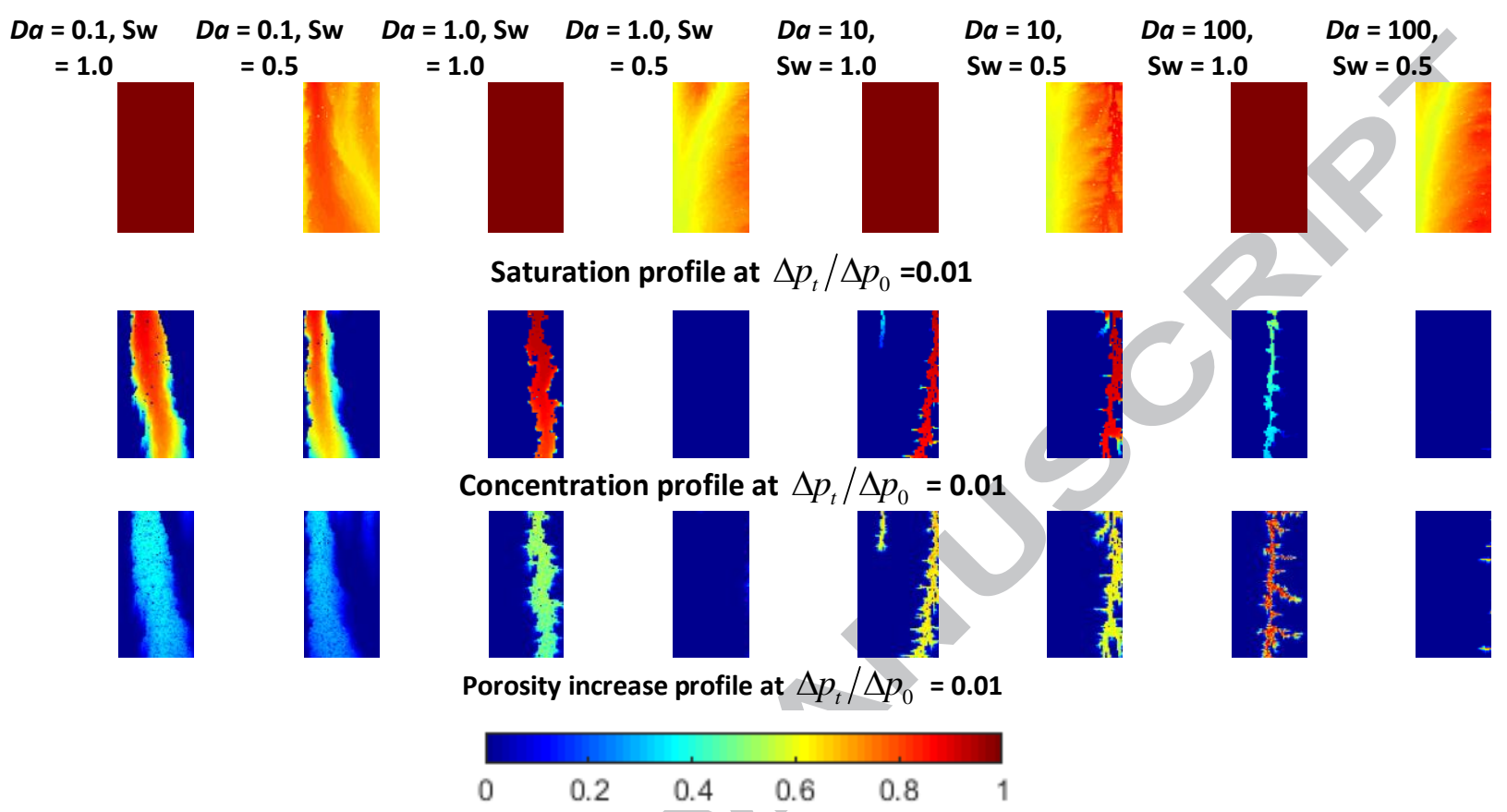

Figure 5 - The profiles of saturation, concentration and porosity increase at the PVI with which $\Delta p_{t} / \Delta p_{0}=0.01$, obtained from simulation suite 1.

An important observation is that the number of branches of the wormholes is reduced under twophase condition. In order to facilitate a better illustration of the reduction in number of branches, we have magnified the graphs for $D a=100$ and removed the background when porosity difference ( $\left.\phi-\phi_{0}\right)$ is zero in Figure 6(a) and Figure 6(b). Although the wormholes for this graph have been depicted for different amounts of acid injected, it is clear that the FWS condition has resulted in more branching. In other words acid has branched out of the main wormhole so that more of it was needed to reach $\Delta p_{t} / \Delta p_{0}=0.01$ for FWS. Compared to the results of Shukla et al., (2006), we observe a less dramatic change in the wormhole configurations in our simulations than the experiments (Figure 6c). Wormholes are thinner and significantly less branched for two-phase conditions. One reason is that for the case of two-phase conditions, Shukla et al., (2006) reduced the water content to residual water saturation by the injection of nitrogen, whereas in our numerical modelling we have kept $50 \%$ water in the domain. Therefore there is $1 / 2$ to $1 / 3$ less acid required for two-phase conditions in Shukla et al., (2006) experiments than when acid was injected without gas. The difference also can be attributed to the difference is the size of the samples used in our model and also on the criteria used to define $\mathrm{PV}_{\mathrm{BT}}$. Shukla et al., (2006) used a core sample of $15.24 \mathrm{~cm}$ long, while as our model was $50 \mathrm{~cm}$ long. Additionally the core sample used by Shukla et al., (2006) is homogeneous (Texas Cream chalk: no vugs or fractures, composed of greater than $99 \% \mathrm{CaCO}_{3}, \mathrm{~K}=$ $6 \mathrm{mD}$ and $\phi=28 \%$ ). Therefore the wormhole generation is not enhanced by rock heterogeneity and impact of phase saturation is more pronounced. 


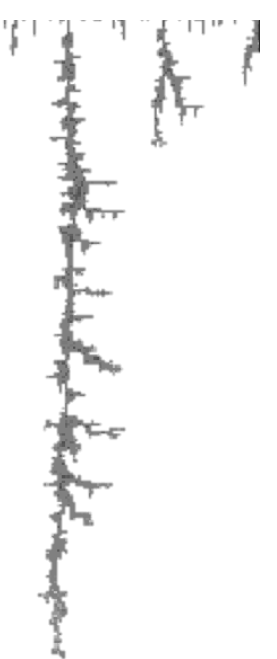

(a) simulation results for $D a=100, S_{w}=1.0$ $P e=2.5 \times 10^{3}$ at $\mathrm{PVI}=5.91$

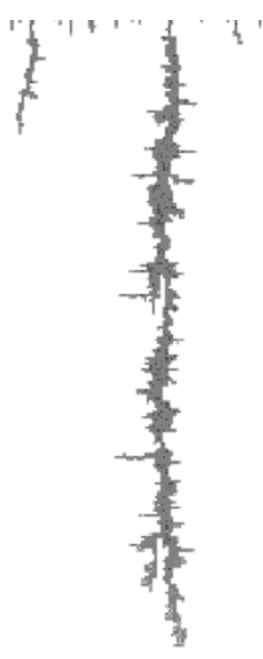

(b) simulation results for $D a=100, S_{w}=0.5$ $P e=2.5 \times 10^{3}$ at $\mathrm{PVI}=4.72$

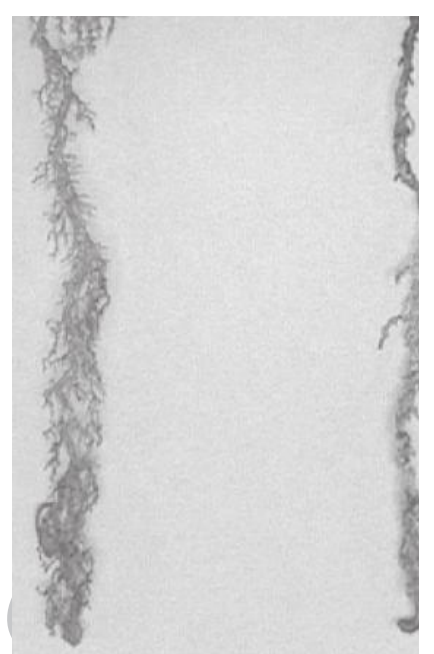

(c) Comparison of wormholes for no gas injection (left) with gas injection prior to acidizing from (right) (Shukla et al., 2006).

Figure 6-Comparison of wormhole configuration between FWS and two-phase conditions.

Figure 7 presents the profiles of pressure gradient reduction for $P e=2.5 \times 10^{5}$ (simulations suite 2). We observe changes in the order of $D a$ regimes in generating effective wormholes compared to $P e=$ $2.5 \times 10^{3}$. By increase in Pe numbers, the optimal $D a$ number has shifted to a range of $D a$ values of approximately 100 to 500 . This is in agreement with experimental results and observations in which for higher Pe numbers (injection rates), higher Da numbers (stronger acids) are required (Fredd and Fogler, 1998a). At this higher injection rate, the flow channels have become more ramified, as fluid is forced into smaller pores.
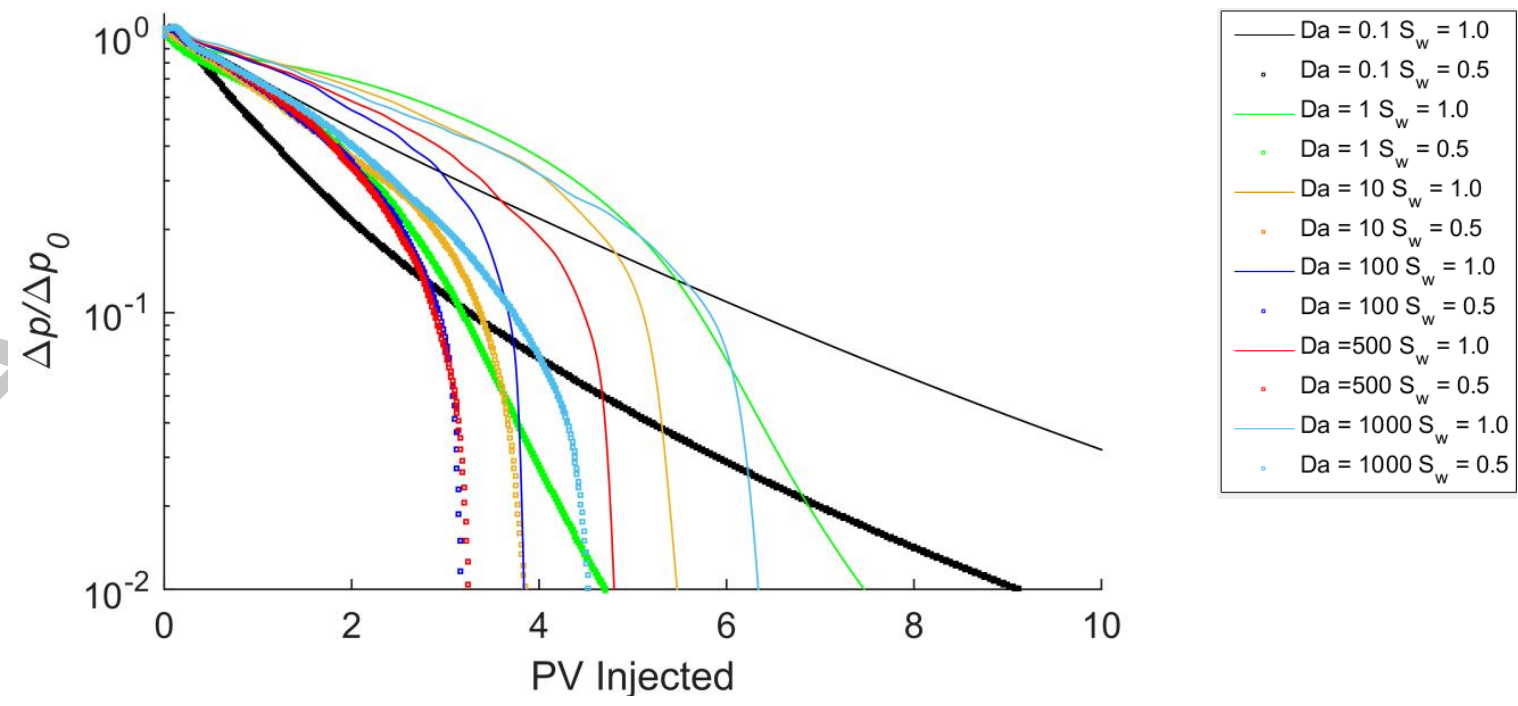

Figure 7 - The pressure gradient reduction vs. initial pore volume injection for suite 2.

Figure 8 shows the profiles of saturation, concentration and porosity increase $\left(\phi-\phi_{0}\right)$ for simulation cases suite 2. A clear difference between the profiles of Figure 5 and Figure 8 are the wormhole configurations and patterns between the two. While in both simulation suites, the pressure gradient 
has been effectively reduced (from 3.83 to 3.17 , equivalent to $17 \%$ decrease) with almost the same $\mathrm{PV}_{\mathrm{BT}}$ of suite 1 , Figure 8 shows wider but less porosity-enhanced wormholes across the domain. In practice, only if we define criteria of wormholing based on the visual characteristics of the wormholes (width, average porosity within the wormholes and its branching), can we distinguish the actual optimal condition of wormhole generation $\left(P e=2.5 \times 10^{3}\right.$ vs. $\left.P e=2.5 \times 10^{5}\right)$.

We repeat the analysis of pressure gradient reduction for lower mobility ratios, where the mobility of water is equal to or less than the mobility of oil. Under the conditions $M<1$, we should expect that the reduced mobility of water will undermine the development and growth of wormholes, since the water phase is less mobile. To investigate this, in Figure 9 for mobility ratio of $M=1$, we show the relative pressure reduction profiles. The results indicate that despite the fact that the mobilities of oil and water are equal, the initial phase saturation is affecting $P V_{B T}$. The difference between $P V_{B T}$ for $D a=0.1,1$ and 10 is large and the difference decreases for $D a=100$ (the optimal $D a$ ). The overall impact of phase saturation is positive for $\mathrm{PV}_{\mathrm{BT}}$ and for the optimal case, $\mathrm{PV} \mathrm{V}_{\mathrm{BT}}$ has reduced from 3.87 to 3.63 ( 6\% decrease).

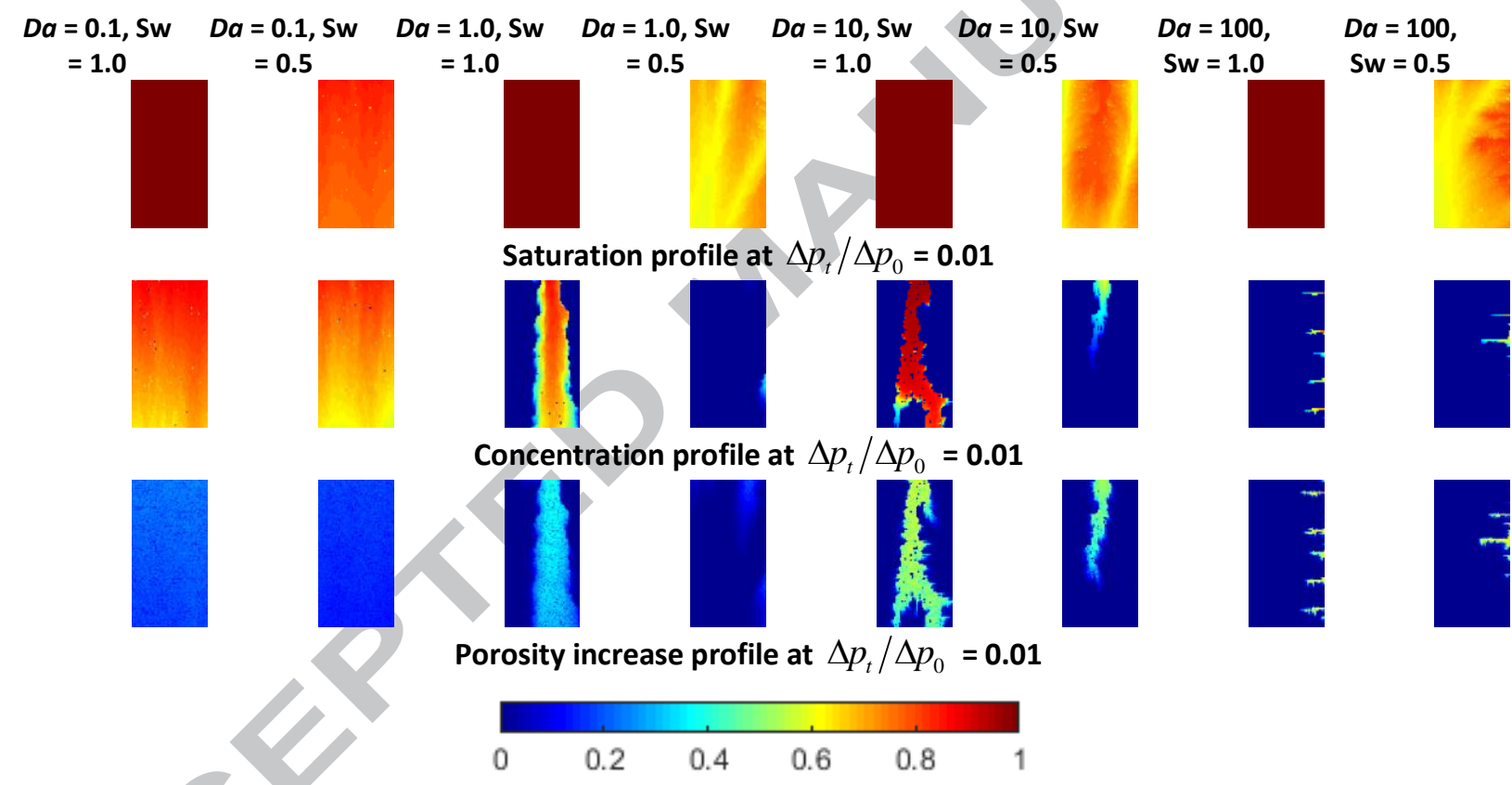

Figure 8 - The profiles of saturation, concentration and porosity increase at the PVI with which $\Delta p_{t} / \Delta p_{0}=0.01$, obtained from simulation suite 2 . 

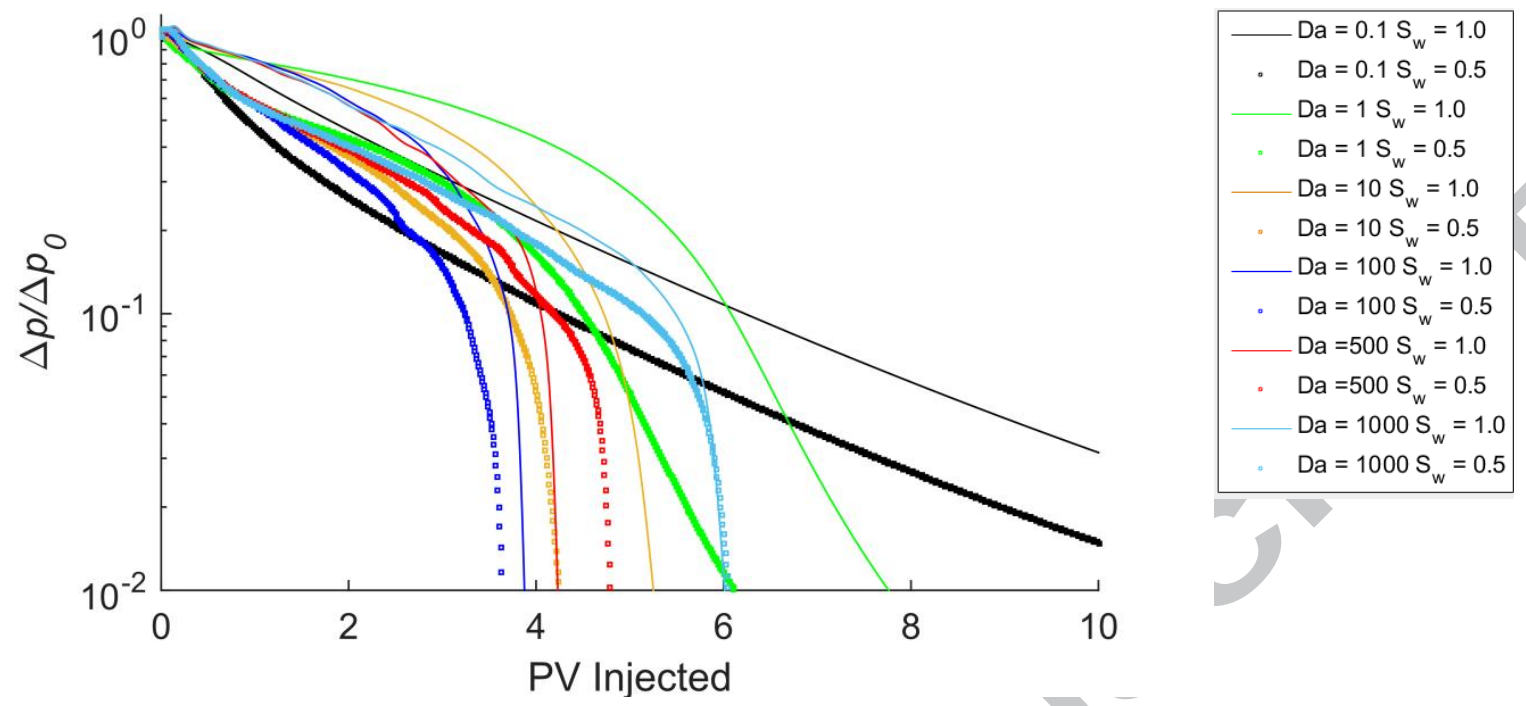

Figure 9 - The pressure gradient reduction vs. initial pore volume injection for suite 3 of simulations.
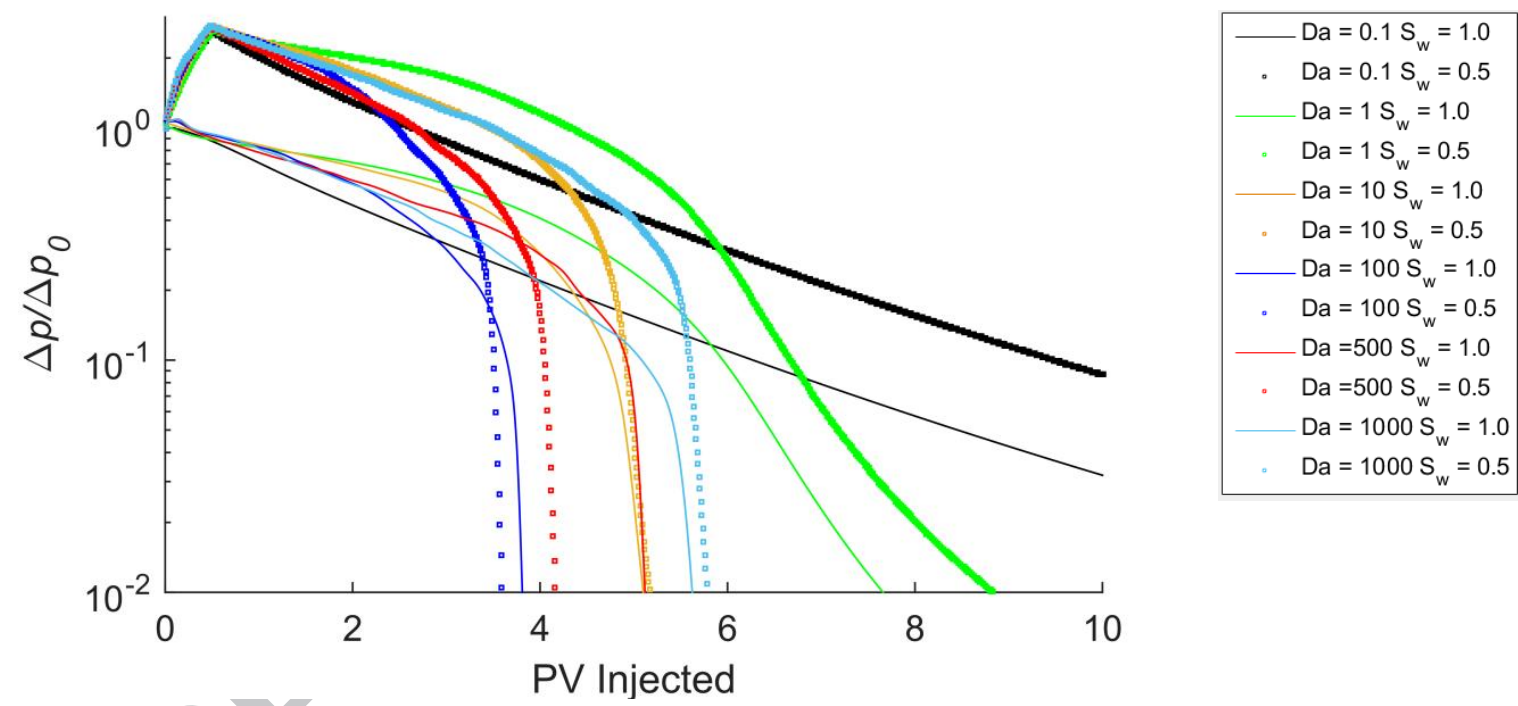

Figure 10-The pressure gradient reduction vs. initial pore volume injection for suite 4 of simulations.

For mobility ratios of $M=0.1$ (i.e., the mobility of water is less than that of the oil and its flow is piston like) in Figure 10, we observe that in presence of initial-oil-in-place saturation, the pressure is initially built up due to resistance of water to flow and later the pressure is released. For several suboptimal $D a$ numbers ( $D a=0.1,1$ and 10$), \mathrm{PV}_{\mathrm{BT}}$ is increased due to presence of initial oil saturation. This can be attributed to a lower mobility of water that is rendering water incapable of breaking through in a non-ideal uniform dissolution pattern. The combination of a piston-like displacement of water and uniform dissolution has generated an adverse condition for the acid to breakthrough. However, interestingly by approaching to the optimal $D a$ of 100 , when the wormholes are generated, we observe that $P V_{B T}$ is reduced from 3.81 to 3.59 ( $6 \%$ decrease). As a result, there are cross-over points in time that the adverse effects of mobility are compensated by the underlying physical mobile-immobile partitioning that we discussed earlier. 


\section{6 - Conclusions and future works}

The development and application of a formulation and computational model for acid rock fracturing under two phase conditions have been presented. This study has demonstrated that a non-zero saturation of an immiscible phase increases the efficiency of the wormholing process (i.e. less volume of acid injection required for wormholes to effectively reduce the pressure gradient), when acid is introduced into a carbonate rock. This numerical study confirms the experimental results of Shukla et al., (2006) and Kumar et al., (2014), that compared with a sample that is initially fully water-saturated, immiscible presence of oil or gas injection prior to acid injection reduces the acid volume needed to propagate the wormholes. Our study shows that the reduction varies between $6 \%$ to $25 \%$ depending on the mobility ratio and Péclet number. Moreover, the wormholes are less branched in the presence of an immiscible phase saturation. The study highlights that different mobility ratios, different initial phase saturation across the domain and different relative permeability curves need to be analysed and simulated for an optimised and efficient matrix acidizing process. A dynamic profile of variations in mobility ratio across the domain can depict how the loss of water phase mobility due to the buffering oil clusters; can lead to faster wormhole generation. The results indicate that a description of the underlying physical reason behind the faster development of wormholes requires an in-depth analysis of pore space partitioning (mobileimmobile zonation) under two-phase condition.

The case study was limited to calcite dissolution since its formulation has been developed more extensively in literature than e.g. the dissolution of sandstones (quartz and silicates) by hydrofluoric acid $\left(4 \mathrm{HF}+\mathrm{SiO}_{2} \rightarrow \mathrm{SiF}_{4}+2 \mathrm{H}_{2} \mathrm{O}\right)$ or mud acid. As detailed in a recent study by Shafiq and Mahmud (2017), a multiple network of reactions occur for sandstone acidizing and as such a more rigorous coupling of geochemistry with flow processes is required. Notwithstanding the fact that, subsurface porous media consist of multiple mineral components such as calcite, clay, quartz, dolomite and pyrite (Noiriel et al. 2007; Noiriel et al., 2013; Chen et al. 2014), a study of the interplay between the immiscible phase and multi-mineral rock (chemically heterogeneous rock) needs to be performed. To this end, multiple reactions have to be considered to take place at different sections or parts of the domain depending on the mineralogical distribution.

From the literature review it is evident that random porosity and permeability distribution is a de facto assumption. Only recently Atchley et al., (2014) and Liu et al., (2016) have considered correlation lengths in permeability and their impact on acidizing performance. By developing a 3D two-phase coupled flow-geochemistry code, the impact of rock heterogeneity needs to be studied in more details in future works.

Finally, while the secondary phase (oil) considered in this work, can be regarded as the insoluble evolved/dissociated $\mathrm{CO}_{2}$ phase from reaction that exceeded the solubility level in the aqueous phase, we have not explicitly labelled the secondary immiscible phase as $\mathrm{CO}_{2}$. In this line of research, the authors have identified the paper by (Bastami and Pourafshary, 2016) who have developed a code for studying the impact of the produced $\mathrm{CO}_{2}$ on acidizing performance. In that paper, when the amount of $\mathrm{CO}_{2}$ present exceeds the solubility value, a $\mathrm{CO}_{2}$ saturation-relative permeability model is evoked, and the relative permeability of the spent acid is reduced. However, the formulation fails to respect a two-phase flow condition where reduction of relative permeability of one phase leads to the reduction of the mobility of that phase and an increase of mobility of the other co-existing phase. As such, extension of the developed code to incorporate the procedure of $\mathrm{CO}_{2}$ production in reaction (as of Bastami and Pourafshary, 2016), its mass transfer through aqueous phase away from 
reacting surface, its dissociation/degassing from aqueous phase when solubility is exceeded, and its flow as a separate secondary phase will be an interesting line of research.

\section{Nomenclature}

Roman letters

\begin{tabular}{|c|c|}
\hline$a_{\mathrm{H}^{+}, \mathrm{b}}$ & activity of $\mathrm{H}^{+}$in bulk of fluid \\
\hline$a_{\mathrm{H}^{+}, \mathrm{s}}$ & activity of $\mathrm{H}^{+}$adjacent to the calcite surface \\
\hline$a_{v}$ & interfacial area per unit volume available for surface reaction \\
\hline$A_{v}$ & dimensionless interfacial area per unit volume available for reaction \\
\hline$a_{0}$ & initial interfacial area per unit volume available for surface reaction \\
\hline$c_{f}$ & dimensionless acid concentration in bulk fluid \\
\hline$C_{f}$ & acid concentration in bulk fluid \\
\hline$c_{s}$ & dimensionless acid concentration at solid-fluid inter \\
\hline$C_{f}$ & acid concentration at solid-fluid interface \\
\hline$C_{\mathrm{H}^{+}, \mathrm{b}}$ & concentration of $\mathrm{H}^{+}$in bulk of fluid \\
\hline$C_{\mathrm{H}^{+}, \mathrm{s}}$ & concentration of $\mathrm{H}^{+}$adjacent to the calcite surface \\
\hline$C_{0}$ & inlet concentration of the acid \\
\hline $\mathbf{D}_{e}$ & effective dispersion tensor \\
\hline$D_{m}$ & molecular diffusion coefficien \\
\hline$D a$ & Damköhler number \\
\hline$f_{\alpha}$ & fractional flow of phase $\alpha$ \\
\hline$h_{T}^{2}$ & pore-scale Thiele modulus \\
\hline$H_{T}^{2}$ & macro-scale Thiele modulus \\
\hline $\mathrm{K}$ & permeability tensor \\
\hline $\mathrm{K}_{0}$ & initial average permeability of rock \\
\hline$k_{c}$ & mass-transfer coefficient \\
\hline$k_{r \alpha}$ & relative permeability of phase $\alpha$ \\
\hline$k_{r \alpha}^{0}$ & end-point relative permeability multiplier for phase $\alpha$ \\
\hline$k_{s}$ & surface reaction rate constant \\
\hline$L$ & length of the core \\
\hline$M$ & mobility ratio \\
\hline$N_{a c}$ & acid capacity number \\
\hline$n$ & experimental exponent for calcite dissolution rate \\
\hline$n_{\alpha}$ & empirical exponent of relative permeability curve for phase $\alpha$ \\
\hline$o$ & oil phase \\
\hline$P$ & pressure \\
\hline$p$ & dimensionless pressure \\
\hline$P_{\text {out }}$ & pressure at the exit boundary of the core \\
\hline$P e$ & Péclet number \\
\hline$P e_{L}$ & longitudinal Péclet number \\
\hline$P e_{T}$ & transversal Péclet number \\
\hline$P V_{B T}$ & pore volumes required to breakthrough \\
\hline$r$ & dimensionless mean pore radius \\
\hline$r_{p}$ & mean pore radius \\
\hline
\end{tabular}


$r_{0}$

$R_{t}$

$R_{s}$

$R e$

$S_{\alpha}$

Sc

Sh

$S h_{\infty}$

$S_{\text {or }}$

$S_{w c}$

$S_{w n}$

$t^{*}$

$t$

$\mathbf{u}_{t}$

$\mathbf{U}_{t}$

$\mathbf{U}_{\alpha}$

$u_{0}$

$w$

$x^{*}$

$y$

$x$

$y^{*}$

Greek letters

$\alpha$

$\alpha_{0 s}$

$\beta$

$\beta_{0,1,2,3}$

$\Delta p_{t}$

$\Delta p_{0}$

$\varepsilon$

$\gamma_{\mathrm{H}^{+}, \mathrm{s}}$

$\kappa$

$\lambda$

$\Lambda_{t}$

$\Lambda_{\alpha}$

$\Lambda_{0}$

$\eta$

$\mu_{\alpha}$

$\xi_{X}$

$\xi_{T}$

$\rho_{s}$

$\phi$

$\phi_{0}$

\section{Acknowledgements}

oil saturation

time

initial mean pore radius

mass transfer rate

surface reaction rate

Reynolds number

saturation of phase $\alpha$

Schmidt number

Sherwood number

asymptotic value of Sherwood number

residual oil saturation

connate water saturation

normalised water saturation with respect to connate water saturation and residual

dimensionless time

dimensionless total velocity

total velocity

velocity of phase $\alpha$

inlet (injection) velocity

water phase

axial/flow direction

transverse directions

dimensionless axial/flow direction

dimensionless transverse directions

dissolving power of acid

coefficient of molecular diffusion in diffusion dominant regimes

pore broadening parameter

fitting parameter for Sherwood number correlation

pressure difference across the core at time $t$

initial pressure difference across the core

breakthrough threshold

activity coefficient $\mathrm{H}^{+}$adjacent to the calcite surface

magnitude of dimensionless permeability

dimensionless total mobility

total mobility

mobility of phase $\alpha$

initial total mobility

ratio of mean pore diameter to core length

viscosity of phase $\alpha$

coefficient of longitudinal hydraulic dispersion in advection-controlled regimes

coefficient of transverse hydraulic dispersion in advection-controlled regimes

solid phase density

porosity of the rock

initial mean porosity 
The computational resources were provided by the University of Manchester EPS Teaching and Research Fund 2014. The authors would like to thank this institution. The authors also appreciate the constructive and valuable comments of the anonymous reviewers of the manuscript.

\section{References}

Alkattan, M., Oelkers, E.H., Dandurand, J.-L., Schott, J., 2002. An experimental study of calcite dissolution rates at acidic conditions and $25 \mathrm{C}$ in the presence of $\mathrm{NaPO} 3$ and $\mathrm{MgCl}$. Chemical Geology 190, 291-302.

Atchley, A.L., Navarre-Sitchler, A.K., Maxwell, R.M., 2014. The effects of physical and geochemical heterogeneities on hydro-geochemical transport and effective reaction rates. Journal of Contaminant Hydrology 165, 53-64.

Babaei, M., Joekar-Niasar, V., 2016. A transport phase diagram for pore-level correlated porous media. Advances in Water Resources 92, 23-29.

Babaei, M., King, P.R., 2012. A modified nested-gridding for upscaling-downscaling in reservoir simulation. Transport in Porous Media 93, 753-775.

Babaei, M., King, P.R., 2013. An upscaling-static-downscaling scheme for simulation of enhanced oil recovery processes. Transport in Porous Media 98, 465-484.

Balakotaiah, V., West, D.H., 2002. Shape normalization and analysis of the mass transfer controlled regime in catalytic monoliths. Chemical Engineering Science 57, 1269-1286.

Bastami, A., Pourafshary, P., 2016. Development of a new model for carbonate matrix acidizing to consider the effects of spent acid. Journal of Energy Resources Technology 138, 052905.

Chen, J.-S., Liu, C.-W., Lai, G.-X., Ni, C.-F., 2009. Effects of mechanical dispersion on the morphological evolution of a chemical dissolution front in a fluid-saturated porous medium. Journal of Hydrology 373, 96-102.

Chen, L., Kang, Q., Robinson, B.A., He, Y.-L., Tao, W.-Q., 2013. Pore-scale modeling of multiphase reactive transport with phase transitions and dissolution-precipitation processes in closed systems. Physical Review E 87, 043306.

Chen, L., Kang, Q., Viswanathan, H.S., Tao, W.Q., 2014. Pore-scale study of dissolution- induced changes in hydrologic properties of rocks with binary minerals, Water Resources Research, 50, 9343-9365.

Cohen, C.E., Ding, D., Quintard, M., Bazin, B., 2008. From pore scale to wellbore scale: Impact of geometry on wormhole growth in carbonate acidization. Chemical Engineering Science 63, 30883099.

Compton, R.G., Pritchard, K.L., Unwin, P.R., 1989. The dissolution of calcite in acid waters: mass transport versus surface control. Freshwater Biology 22, 285-288.

Cubillas, P., Köhler, S., Prieto, M., Causserand, C., Oelkers, E.H., 2005. How do mineral coatings affect dissolution rates? An experimental study of coupled $\mathrm{CaCO} 3$ dissolution-CdCO3 precipitation. Geochimica et Cosmochimica Acta 69, 5459-5476. 
Daccord, G., Lenormand, R., Lietard, O., 1993. Chemical dissolution of a porous medium by a reactive fluid-I. Model for the "wormholing" phenomenon. Chemical Engineering Science 48, 169-178.

Daccord, G., Touboul, E., Lenormand, R., 1989. Carbonate acidizing: toward a quantitative model of the wormholing phenomenon. Society of Petroleum Engineers 4, 63-68.

Davies, S., Kelkar, S., 2007. Carbonate Stimulation, Schlumberger Middle East \& Asia Reservoir Review Number 8.

Davis, M.E., Davis, R.J., 2012. Fundamentals of chemical reaction engineering. Chapter 6 - Effects of Transport Limitations on Rates of Solid-Catalyzed Reactions, Courier Corporation.

De Gennes, P.G., 1983. Hydrodynamic dispersion in unsaturated porous media. Journal of Fluid Mechanics 136, 189-200.

De Smedt, F. d, Wierenga, P.J., 1984. Solute transfer through columns of glass beads. Water Resources Research 20, 225-232.

Evans, R.L., Lizarralde, D., 2003. Geophysical evidence for karst formation associated with offshore groundwater transport: an example from North Carolina. Geochemistry, Geophysics, Geosystems 4.

Fredd, C.N., 2000. Dynamic model of wormhole formation demonstrates conditions for effective skin reduction during carbonate matrix acidizing, in: SPE Permian Basin Oil and Gas Recovery Conference.

Fredd, C.N., Fogler, H.S., 1998a. Influence of transport and reaction on wormhole formation in porous media. AIChE journal 44, 1933-1949.

Fredd, C.N., Fogler, H.S., 1998b. The kinetics of calcite dissolution in acetic acid solutions. Chemical Engineering Science 53, 3863-3874.

Fu, X., Cueto-Felgueroso, L., Bolster, D., Juanes, R., 2015. Rock dissolution patterns and geochemical shutdown of $\mathrm{CO}_{2}$-brine-carbonate reactions during convective mixing in porous media. Journal of Fluid Mechanics 764, 296-315.

Ghommem, M., Zhao, W., Dyer, S., Qiu, X., Brady, D., 2015. Carbonate acidizing: Modeling, analysis, and characterization of wormhole formation and propagation. Journal of Petroleum Science and Engineering 131, 18-33.

Glasbergen, G., Kalia, N., Talbot, M.S., 2009. The Optimum Injection Rate for Wormhole Propagation: Myth or Reality?, in: 8th European Formation Damage Conference.

Golfier, F., Bazin, B., Zarcone, C., Lernormand, R., Lasseux, D., Quintard, M., 2001. Acidizing carbonate reservoirs: numerical modelling of wormhole propagation and comparison to experiments, in: SPE European Formation Damage Conference.

Golfier, F., Quintard, M., Bazin, B., Lenormand, R., 2006. Core-scale description of porous media dissolution during acid injection-part II: calculation of the effective properties. Computational \& Applied Mathematics 25, 55-78. 
Hung, K.M., Hill, A.D., Sepehrnoori, K., 1989. A mechanistic model of wormhole growth in carbonate matrix acidizing and acid fracturing. Journal of Petroleum Technology 41, 59-66.

Islam, A., Korrani, A.K.N., Sepehrnoori, K., Patzek, T., 2014. Effects of geochemical reaction on double diffusive natural convection of $\mathrm{CO}_{2}$ in brine saturated geothermal reservoir. Energy Procedia 63, 5357-5377.

Islam, A., Sun, A., Lu, J., 2016. Simulating in-zone chemistry changes from injection time to longer periods of $\mathrm{CO}_{2}$ storage. Environmental Earth Sciences 75, 1346.

Johnson, J.W., Nitao, J.J., Knauss, K.G., 2004. Reactive transport modeling of $\mathrm{CO}_{2}$ storage in saline aquifers to elucidate fundamental processes, trapping mechanisms and sequestration partitioning. Geological Storage of Carbon Dioxide 233, 107-128.

Kalia, N., Balakotaiah, V., 2007. Modeling and analysis of wormhole formation in reactive dissolution of carbonate rocks. Chemical Engineering Science 62, 919-928.

Kalia, N., Balakotaiah, V., 2009. Effect of medium heterogeneities on reactive dissolution of carbonates. Chemical Engineering Science 64, 376-390.

Kampman, N., Bickle, M., Wigley, M., Dubacq, B., 2014. Fluid flow and $\mathrm{CO}_{2}$-fluid-mineral interactions during $\mathrm{CO}_{2}$-storage in sedimentary basins. Chemical Geology 369, 22-50.

Karadimitriou, N.K., Joekar-Niasar, V., Babaei, M., Shore, C.A., 2016. The critical role of immobile zone in non-Fickian two-phase transport: A new paradigm. Environmental Science \& Technology $50,4348-4392$.

Kumar, R., He, J., Nasr-El-Din, H., 2014. Effect of oil saturation on acid propagation during matrix acidization of carbonate rocks, in: SPE Latin America and Caribbean Petroleum Engineering Conference.

Liu, M., Mostaghimi, P., 2017a. Characterisation of reactive transport in pore-scale correlated porous media. Chemical Engineering Science 173, 121-130.

Liu, M., Mostaghimi, P., 2017b. High-resolution pore-scale simulation of dissolution in porous media. Chemical Engineering Science 161, 360-369.

Liu, M., Zhang, S., Mou, J., Zhou, F., 2013. Wormhole propagation behavior under reservoir condition in carbonate acidizing. Transport in Porous Media 96, 203-220.

Liu, N., Liu, M., 2016. Simulation and analysis of wormhole propagation by VES acid in carbonate acidizing. Journal of Petroleum Science and Engineering 138, 57-65.

Liu, P., Yao, J., Couples, G.D., Ma, J., Huang, Z., Sun, H., 2017. Modelling and simulation of wormhole formation during acidization of fractured carbonate rocks. Journal of Petroleum Science and Engineering.

Liu, P., Xue, H., Zhao, L.Q., Zhao, X.D., Cui, M.Y., 2016. Simulation of 3D multi-scale wormhole propagation in carbonates considering correlation spatial distribution of petrophysical properties. Journal of Natural Gas Science and Engineering 32, 81-94. 
Lund, K., Fogler, H.S., McCune, C.C., 1973. Acidization-I. The dissolution of dolomite in hydrochloric acid. Chemical Engineering Science 28, 691IN1-700.

Maheshwari, P., Balakotaiah, V., 2013. 3D Simulation of carbonate acidization with $\mathrm{HCl}$ : comparison with experiments, in: SPE Production and Operations Symposium.

Maheshwari, P., Ratnakar, R.R., Kalia, N., Balakotaiah, V., 2013. 3-D simulation and analysis of reactive dissolution and wormhole formation in carbonate rocks. Chemical Engineering Science 90, 258-274.

Morse, J.W., Arvidson, R.S., 2002. The dissolution kinetics of major sedimentary carbonate minerals. Earth-Science Reviews 58, 51-84.

$\mathrm{Na}$, J., Xu, T., Jiang, Z., Bao, X., Yongdong, W., Feng, B., 2016. A study on the interaction of mud acid with rock for chemical stimulation in an enhanced geothermal system. Environmental Earth Sciences 75, 1-13.

Nick, H.M., Raoof, A., Centler, F., Thullner, M., Regnier, P., 2013. Reactive dispersive contaminant transport in coastal aquifers: Numerical simulation of a reactive Henry problem. Journal of Contaminant Hydrology 145, 90-104.

Nierode, D.E., Williams, B.R., Bombardieri, C.C., 1972. Prediction of stimulation from acid fracturing treatments. Journal of Canadian Petroleum Technology 11.

Noiriel, C., Madé, B., Gouze, P., 2007. Impact of coating development on the hydraulic and transport properties in argillaceous limestone fracture, Water Resources Research 43.

Noiriel, C., Gouze, P., Madé, B., 2013. 3D analysis of geometry and flow changes in a limestone fracture during dissolution, Journal of Hydrology 486, 211-223.

Ott, H., Oedai, S., Pentland, C.H., Eide-Engdahl, K., van der Linden, A.J., Gharbi, O., Bauer, A., Makurat, A., 2013. $\mathrm{CO}_{2}$ reactive transport in limestone: flow regimes, fluid flow and mechanical rock properties, in: International Symposium of the Society of Core Analysts. Napa Valley, California, USA. pp. 16-19.

Panga, M.K.R., Ziauddin, M., Balakotaiah, V., 2005. Two-scale continuum model for simulation of wormholes in carbonate acidization. AIChE Journal 51, 3231-3248.

Parmigiani, A., Huber, C., Bachmann, O., Chopard, B., 2011. Pore-scale mass and reactant transport in multiphase porous media flows. Journal of Fluid Mechanics 686, 40-76.

Plummer, L.N., Wigley, T.M.L., Parkhurst, D.L., 1978. The kinetics of calcite dissolution in $\mathrm{CO}_{2}$-water systems at 5 degrees to 60 degrees $\mathrm{C}$ and 0.0 to $1.0 \mathrm{~atm} \mathrm{CO}_{2}$. American Journal of Science 278, 179-216.

Portier, S., André, L., Vuataz, F.-D., 2007. Review on chemical stimulation techniques in oil industry and applications to geothermal systems, Technical Report, Centre for Geothermal Research, Neuchâtel, Switzerland.

Portier, S., Vuataz, F.D., 2010. Developing the ability to model acid-rock interactions and mineral dissolution during the RMA stimulation test performed at the Soultz-sous-Forêts EGS site, France. 
Comptes Rendus Geoscience 342, 668-675.

Qin, X.S., Huang, G.H., Chakma, A., Chen, B., Zeng, G.M., 2007. Simulation-based process optimization for surfactant-enhanced aquifer remediation at heterogeneous DNAPLcontaminated sites. Science of the Total Environment 381, 17-37.

Qiu, X., Zhao, W., Chang, F., Dyer, S., 2013. Quantitative Modeling of Acid Wormholing in Carbonates-What Are the Gaps to Bridge, in: SPE Middle East Oil and Gas Show and Conference.

Rochelle, C.A., Czernichowski-Lauriol, I., Milodowski, A.E., 2004. The impact of chemical reactions on $\mathrm{CO}_{2}$ storage in geological formations: a brief review. Geological Society, London, Special Publications 233, 87-106.

Schechter, R.S., Gidley, J.L., 1969. The change in pore size distribution from surface reactions in porous media. AIChE Journal 15, 339-350.

Sedighi, M., Thomas, H.R., Al Masum, S., Vardon, P.J., Nicholson, D., Chen, Q., 2015. Geochemical modelling of hydrogen gas migration in an unsaturated bentonite buffer. Geological Society, London, Special Publications 415, 189-201.

Sedighi, M., Thomas, H.R., Vardon, P.J., 2016. Reactive transport of chemicals in unsaturated soils: numerical model development and verification. Canadian Geotechnical Journal 53, 162-172.

Shafiq, M.U., Mahmud, H.B., 2017. Sandstone matrix acidizing knowledge and future development. Journal of Petroleum Exploration and Production Technology 1-12.

Shukla, S., Zhu, D., Hill, A.D., 2006. The effect of phase saturation conditions on wormhole propagation in carbonate acidizing. SPE Journal 11, 273-281.

Sjöberg, E.L., Rickard, D., 1983. The influence of experimental design on the rate of calcite dissolution. Geochimica et Cosmochimica Acta 47, 2281-2285.

Spycher, N.F., Sonnenthal, E.L., Apps, J.A., 2003. Fluid flow and reactive transport around potential nuclear waste emplacement tunnels at Yucca Mountain, Nevada. Journal of Contaminant Hydrology 62, 653-673.

Steefel, C.I., Lasaga, A.C., 1994. A coupled model for transport of multiple chemical species and kinetic precipitation/dissolution reactions with application to reactive flow in single phase hydrothermal systems. American Journal of science 294, 529-592.

Valdes-Abellan, J., Jiménez-Martinez, J., Candela, L., Jacques, D., Kohfahl, C., Tamoh, K., 2017. Reactive transport modelling to infer changes in soil hydraulic properties induced by nonconventional water irrigation. Journal of Hydrology 549, 114-124.

Wyllie, M.R.J., Gardner, G.H.F., 1958. The generalized Kozeny-Carman equation. World oil 146, 121128.

Xiong, Y., Fakcharoenphol, P., Winterfeld, P., Zhang, R., Wu, Y.-S., 2013. Coupled geomechanical and reactive geochemical model for fluid and heat flow: application for enhanced geothermal reservoir, in: SPE Reservoir Characterization and Simulation Conference and Exhibition. 
Zhao, C., Hobbs, B., Ord, A., 2013. Theoretical analyses of acidization dissolution front instability in fluid-saturated carbonate rocks. International Journal for Numerical and Analytical Methods in Geomechanics 37, 2084-2105. 

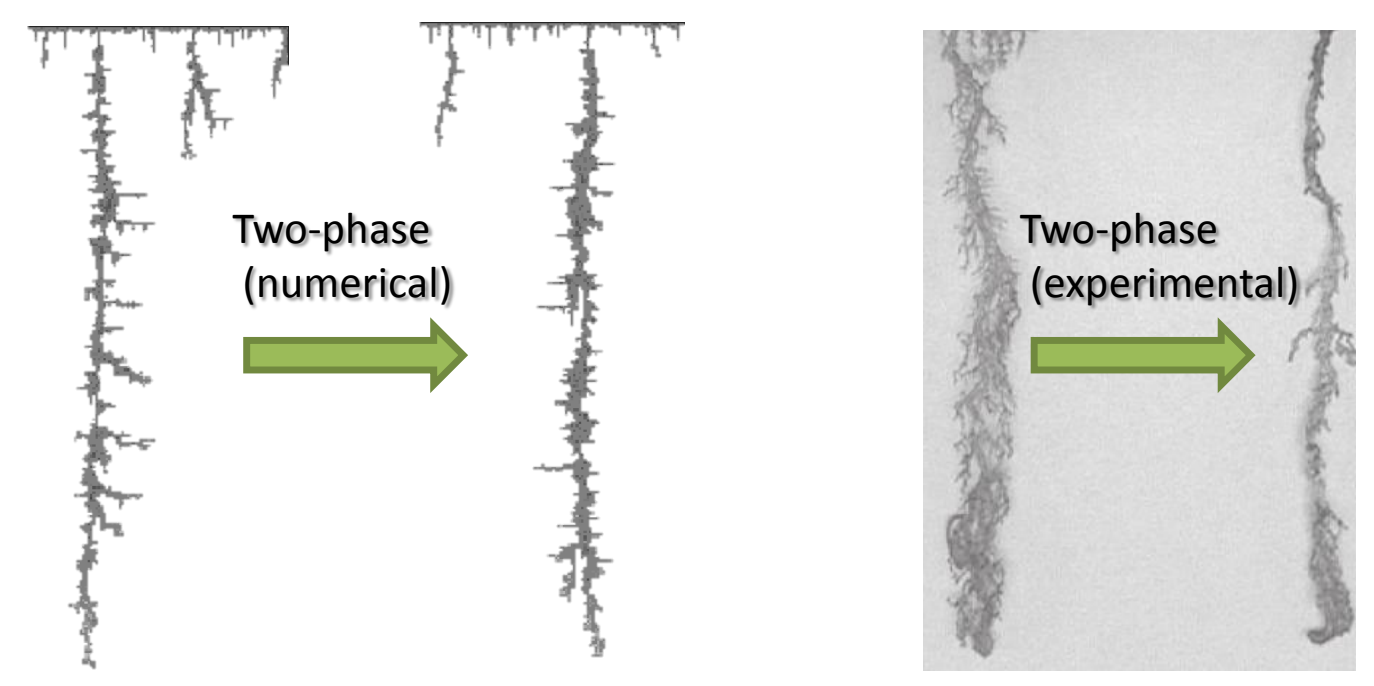

Two-phase

(experimental) 


\section{Highlights:}

- Numerical modelling of carbonate matrix acidizing under two phase conditions developed

- Impact of phase saturation and varying mobility ratio investigated

- In the presence of immiscible phase (such as oil) the wormholes more efficiently produced

- The amount of acid required for reducing pressure decreased under two-phase conditions 